\title{
Long-range correction for tight-binding TD-DFT
}

\author{
Alexander Humeniuk and Roland Mitrić* \\ Institut für Physikalische und Theoretische Chemie, Julius-Maximilians Universität Würzburg, \\ Emil-Fischer-Straße 42, 97074 Würzburg \\ E-mail: roland.mitric@uni-wuerzburg.de
}

\begin{abstract}
We present two improvements to the tight-binding approximation of time-dependent density functional theory (TD-DFTB): Firstly, we add an exact Hartree-Fock exchange term, which is switched on at large distances, to the ground state Hamiltonian and similarly to the coupling matrix that enters the linear response equations for the calculation of excited electronic states. We show that the excitation energies of charge transfer states are improved relative to the standard approach without the long-range correction by testing the method on a set of molecules from the database in [J. Chem. Phys. 2008, 128, 044118] that are known to exhibit problematic charge transfer states. The degree of spatial overlap between occupied and virtual orbitals indicates where TD-DFTB and lc-TD-DFTB can be expected to produce large errors.

Secondly, we improve the calculation of oscillator strengths. The transition dipoles are obtained from Slater Koster files for the dipole matrix elements between valence orbitals. In particular, excitations localized on a single atom, which appear dark when using Mulliken transition charges, in this way acquire a more realistic oscillator strength.

These extensions pave the way for using long-range corrected TD-DFTB (lc-TD-DFTB) to describe the electronic structure of large chromophoric polymers, where uncorrected TD-
\end{abstract}

\footnotetext{
${ }^{*}$ To whom correspondence should be addressed
} 
DFTB fails to describe the high degree of conjugation and produces spurious low-lying charge transfer states.

\section{Introduction}

Tight-binding DFT (DFTB) $)^{\sqrt[2]{4}}$ and its time-dependent formulation TD-DFTB ${ }^{[56}$ are semi-empirical methods based on (TD)-DFT ${ }^{7}-9$ that inherit many of the advantages and shortcomings of the latter. The failure of TD-DFT to describe charge transfer states ${ }^{10}$ is particularly severe if one deals with extended molecules or oligomers with a large degree of conjugation. These charge transfer states, which appear at unphysically low energies, can be removed if a long-range exchange term is included, leading to the long-range corrected TD-DFT. 11

In the tight-binding formulation this correction can also be included at almost no additional computational cost. The possibility to include a range separated functional into DFTB has been explored before by Niehaus and Della Sala. ${ }^{12}$ They generate the pseudo atom basis starting from a full DFT calculation on a single atom with a range-separated functional. This changes the electronic parametrization and makes the pseudoatoms depend on the long-range correction. Then they consistently add the first order correction arising from the long-range correction in the tightbinding Kohn-Sham equations and the linear response formalism.

Here instead, a much simpler (maybe less rigorous) approach is proposed, where the existing electronic parameters (pseudo atoms and Hubbard parameters) are left untouched. Since the usual DFTB parametrizations are based on a local density approximation (LDA), the long range exchange can be incorporated by simply adding the attenuated exact exchange energy $E_{x}^{\mathrm{lr}}$ to the total electronic ground state energy. For the calculation of excited states, a long range correction term is added to the coupling matrix in the spirit of CAM-B3LYP.13 The exchange integrals are then approximated by products of transition charges as usual. The distance at which the exact exchange is gradually switched on is controlled by new a parameter $R_{\mathrm{lr}}$ (equivalent to $1 / \mu$ in the notation of ref. ${ }^{11}$ ) that can be adjusted to fit excitation energies to CAM-B3LYP results or experimental 
values.

This article is structured as follows: In sections 2.1 and 2.2 the working formulae for the longrange correction and the transition dipoles are derived. Section 2.3 deals with different ways of quantifying charge transfer at the level of tight-binding DFT. Finally the method is applied to a test set of molecules and compared to CAM-B3LYP results in sections 3 and 4 .

Technical details are deferred to the appendices:

- Appendix A elucidates ambiguities in the definition of the $\gamma$-matrix that lead to differences between DFTB implementations.

- Appendix $\mathrm{B}$ discusses different ways of splitting the Coulomb potential into a short and a long range part.

- Slater-Koster rules for transition dipoles are derived in the appendices C, D and E.

\section{Method Description}

Many review articles have been written about DFTB, ${ }^{14 \mid 15}$ for a pedagogical introduction see. ${ }^{3 /}$ To make clear at which points we introduce modifications, we recapitulate here the basics of DFTB and TD-DFTB:

To derive the DFTB Hamiltonian, one starts with the full DFT energy functional 16

$$
E[\rho]=\sum_{\alpha} f_{a}\left\langle\phi_{\alpha}\left|\left(-\frac{1}{2} \nabla^{2}+\int V_{\mathrm{ext}} \rho\right)\right| \phi_{\alpha}\right\rangle+\frac{1}{2} \iint^{\prime} \frac{\rho \rho^{\prime}}{\left|\vec{r}-\vec{r}^{\prime}\right|}+E_{\mathrm{xc}}[\rho]+E_{\mathrm{nuc}}
$$

where $\phi_{\alpha}$ are the Kohn-Sham orbitals of the system of non-interacting electrons with the occupation numbers $f_{\alpha}, V_{\text {ext }}$ is the potential the nuclei exert on the electrons, as well as any external electric field, $E_{x c}$ is the exchange-correlation functional and $E_{\text {nuc }}$ the energy due to Coulomb repulsion between nuclei. One proceeds by expanding the energy around a reference density, $\rho=\rho_{0}+\delta \rho$, to second order in $\delta \rho$. The reference density $\rho_{0}$ is a superposition of atomic densities of the individually neutral atoms, while the redistribution of the electron density $\delta \rho$ is a result of the chemical 
bonding.

After rearranging the expansion of the energy functional,

$$
\begin{aligned}
E\left[\rho_{0}+\delta \rho\right] \approx & \sum_{\alpha} f_{\alpha}\left\langle\phi_{\alpha}\left|\left(-\frac{1}{2} \nabla^{2}+V_{\mathrm{ext}}+V_{H}\left[\rho_{0}\right]+V_{\mathrm{xc}}\left[\rho_{0}\right]\right)\right| \phi_{\alpha}\right\rangle \\
& +\frac{1}{2} \iint^{\prime}\left(\frac{\delta^{2} E_{x c}\left[\rho_{0}\right]}{\delta \rho \delta \rho^{\prime}}+\frac{1}{\left|\vec{r}-\vec{r}^{\prime}\right|}\right) \delta \rho \delta \rho^{\prime} \\
& -\frac{1}{2} \int V_{H}\left[\rho_{0}\right] \rho_{0}(\vec{r})+\left(E_{x c}\left[\rho_{0}\right]-\int V_{x c}\left[\rho_{0}\right] \rho_{0}(\vec{r})\right)+E_{\mathrm{nuc}}
\end{aligned}
$$

the energy is partitioned into terms depending on the reference density and the orbitals $\left(E_{\mathrm{bs}}\right)$, the density fluctuations $\left(E_{\text {coul,xc }}\right)$ and the repulsive energy $\left(E_{\text {rep }}\right)$, which stands for everything else not covered by the first two terms:

$$
\begin{aligned}
E\left[\rho_{0}+\delta \rho\right] \approx & \sum_{a} f_{a}\left\langle\phi_{a}\left|H\left[\rho_{0}\right]\right| \phi_{a}\right\rangle & & \text { band structure energy } E_{b s} \\
& +\frac{1}{2} \iint^{\prime}\left(\frac{\delta^{2} E_{x c}\left[\rho_{0}\right]}{\delta \rho \delta \rho^{\prime}}+\frac{1}{\left|\vec{r}-\vec{r}^{\prime}\right|}\right) \delta \rho \delta \rho^{\prime} & & \text { Coulomb + part of xc energy } E_{\mathrm{coul}, \mathrm{xc}} \\
& +E_{\text {nuc }}+\text { everything else } & & \text { repulsive energy } E_{\mathrm{rep}}
\end{aligned}
$$

To approximate $E_{\text {coul,xc }}$ one assumes that the charge fluctuation $\delta \rho$ can be decomposed into spherically symmetric contributions centered on the atoms, $\delta \rho=\sum_{I}^{N_{a t}} \Delta q_{I} F_{I}\left(\left|\vec{r}-\vec{R}_{I}\right|\right)$, where $\Delta q_{I}$ are the excess Mulliken charges on atom I:

$$
\begin{aligned}
E_{\mathrm{coul}, \mathrm{xc}}\left[\rho_{0}+\delta \rho\right] & =\frac{1}{2} \iint^{\prime}\left(\frac{\delta^{2} E_{x c}\left[\rho_{0}\right]}{\delta \rho \delta \rho^{\prime}}+\frac{1}{\left|\vec{r}-\vec{r}^{\prime}\right|}\right) \delta \rho \delta \rho^{\prime} \\
& =\sum_{I}^{N_{a t}} \sum_{J}^{N_{a t}} E_{\mathrm{coul}, \mathrm{xc}}^{I J}
\end{aligned}
$$

For partial charges sitting on different atoms, $I \neq J$, only the electrostatic interaction is taken into account (depending on the distance $R_{I J}$ of the atomic centers) and any exchange or correlation interaction is neglected, since the exchange correlation functional is assumed to be local. The interaction energy of charge with itself on the same atom is controlled by the Hubbard parameters 
$U_{H}$, which can be obtained from experimental ionization energies and electron affinities, or abinitio calculations. This leads to a partitioning of $E_{\mathrm{coul}, \mathrm{xc}}^{I J}$ into pievewise contributions:

$$
E_{\mathrm{coul}, \mathrm{xc}}^{I J}=\left\{\begin{array}{ll}
\frac{1}{2} \Delta q_{I} \Delta q_{J} \iint^{\prime} \frac{F_{I} F_{J}^{\prime}}{\left|\vec{r}-\vec{r}^{\prime}\right|} & I \neq J \\
\frac{1}{2} U_{H}\left(\Delta q_{I}\right)^{2} & I=J
\end{array}\right\}=\frac{1}{2} \Delta q_{I} \Delta q_{J} \gamma_{I J}\left(R_{I J}\right)
$$

The approximate DFTB energy is now a function of the partial Mulliken charges instead of the density:

$$
E_{\mathrm{DFTB}}\left[\left\{\Delta q_{I}\right\}\right]=\sum_{\alpha} f_{\alpha}\left\langle\phi_{\alpha}\left|H\left[\rho_{0}\right]\right| \phi_{a}\right\rangle+\frac{1}{2} \sum_{I J} \gamma_{I J}\left(R_{I J}\right) \Delta q_{I} \Delta q_{J}+\underbrace{\left(E[\rho]-E_{\mathrm{DFTB}}\right)}_{\sum_{I<J} V_{\mathrm{rep}}^{I J}\left(R_{I J}\right)}
$$

All the deviations from the true energy are absorbed into the repulsive potential, which ideally should only depend on the molecular geometry but not on the charge distribution. In a rough approximation, these deviations can be decomposed into contributions from pairs of atoms, $V_{\text {rep }}^{I J}\left(R_{I J}\right)$, and be adjusted to higher-level DFT methods.

Fitting ${ }^{17}$ and validating ${ }^{18}$ the repulsive potentials is the most time-consuming part of parametrizing the DFTB method. Although properly adjusted repulsive potentials are crucial for molecular dynamics simulations, structure optimization or vibrational spectra, we will neglect them here, as they have no influence on the electronic absorption spectra.

The Kohn-Sham orbitals are expanded into a minimal set of pseudo-atomic orbitals $\{|\mu\rangle\}$, which are compressed by a confining potential, since orbitals in a molecule are less diffuse than in the free atoms:

$$
\left|\phi_{i}\right\rangle=\sum_{\mu} c_{\mu}^{i}|\mu\rangle
$$

A variation of the energy with respect to the Kohn-Sham orbitals, under the constraint that the orbitals are normalized, leads to the DFTB equivalent of the Kohn-Sham equations with the DFTB 
Hamiltonian (in the basis of atomic orbitals $\mu$ and $v$ ):

$$
H_{\mu \nu}^{\mathrm{DFTB}}=\underbrace{\left\langle\mu\left|H\left[\rho_{0}\right]\right| v\right\rangle}_{H_{\mu \nu}^{0}}+\frac{1}{2} \underbrace{\langle\mu \mid v\rangle}_{S_{\mu \nu}} \sum_{K=1}^{N_{a t}}\left(\gamma_{I K}+\gamma_{J K}\right) \Delta q_{K} \quad \mu \in I, v \in J
$$

where $\mu \in I$ means that the atomic orbital $\mu$ belongs to atom $I$.

The Kohn-Sham equations are solved self-consistently: In each step the new partial Mulliken charges $\Delta q_{I}$ and the new Hamiltonian are computed from the orbital coefficients of the previous iteration and the resulting Kohn-Sham equations are solved to give the next orbital coefficients. These steps are repeated until the charge distribution and the density matrix do not change anymore.

Excited states are calculated in the framework of linear-response TD-DFT, which was adapted to tight-binding DFT by Niehaus. ${ }^{[5]}$ For brevity, we only sketch the working equations of TDDFT and how they are adapted to the tight-binding formulation. A converged DFT calculation provides the single-particle Kohn-Sham orbitals, and to a first approximation excitation energies are differences of virtual and occupied Kohn-Sham energies $\omega_{o v \sigma}=\varepsilon_{v \sigma}-\varepsilon_{o \sigma}$.

The coupling matrix

$$
K_{o v \sigma, o^{\prime} v^{\prime} \sigma^{\prime}}=\iint \phi_{o \sigma}\left(\vec{r}_{1}\right) \phi_{v \sigma}\left(\vec{r}_{1}\right)\left[\frac{1}{\left|\vec{r}_{1}-\vec{r}_{2}\right|}+\frac{\delta^{2} E_{x c}}{\delta \rho_{\sigma}\left(\vec{r}_{1}\right) \delta \rho_{\sigma^{\prime}}\left(\vec{r}_{2}\right)}\right] \phi_{o^{\prime} \sigma^{\prime}}\left(\vec{r}_{2}\right) \phi_{v^{\prime} \sigma^{\prime}}\left(\vec{r}_{2}\right) d^{3} r_{1} d^{3} r_{2}
$$

represents the response of the Kohn-Sham potential to a perturbation of the electron density and is responsible for adding a correction to the Kohn-Sham orbital energy differences.

In linear-response TD-DFT excited states are computed from the Hermitian eigenvalue prob$1 \mathrm{em}{ }^{8}$

$$
(\mathbf{A}-\mathbf{B})^{1 / 2}(\mathbf{A}+\mathbf{B})(\mathbf{A}-\mathbf{B})^{1 / 2} \vec{F}_{I}=\Omega_{I}^{2} \vec{F}_{I}
$$


where the matrices $\mathbf{A}$ and $\mathbf{B}$ contain the coupling matrix:

$$
\begin{aligned}
& A_{o v \sigma, o^{\prime} v^{\prime} \sigma^{\prime}}=\delta_{o, o^{\prime}} \delta_{v, v^{\prime}} \delta_{\sigma, \sigma^{\prime}}\left(\varepsilon_{v \sigma}-\varepsilon_{o \sigma}\right)+K_{o v \sigma, o^{\prime} v^{\prime} \sigma^{\prime}} \\
& B_{o v \sigma, o^{\prime} v^{\prime} \sigma^{\prime}}=K_{o v \sigma, v^{\prime} o^{\prime} \sigma^{\prime}}
\end{aligned}
$$

The solution of eqn. (??) provides the excitation energies $E_{I}=\hbar \Omega_{I}$ as eigenvalues and the coefficients for single excitations from occupied to virtual orbitals $(o \rightarrow v)$

$$
\vec{C}_{o v \sigma}^{I}=\frac{1}{\sqrt{\Omega_{I}}} \sum_{o^{\prime} \in o c c} \sum_{v^{\prime} \in v i r t} \sum_{\sigma^{\prime}}\left[(\mathbf{A}-\mathbf{B})^{1 / 2}\right]_{o v \sigma, o^{\prime} v^{\prime} \sigma^{\prime}} \vec{F}_{o^{\prime} v^{\prime} \sigma^{\prime}}^{I}
$$

as eigenvectors.

In the absence of a non-local exchange term, $(\mathbf{A}-\mathbf{B})$ is effectively diagonal so that eqn. (??) can be simplified to yield Casida's equation: $\underline{8}$

$$
\sum_{o^{\prime} \in o c c} \sum_{v^{\prime} \in v i r t} \sum_{\sigma^{\prime}}\left[\delta_{o, o^{\prime}} \delta_{v, v^{\prime}} \delta_{\sigma, \sigma^{\prime}}\left(\varepsilon_{v \sigma}-\varepsilon_{o \sigma}\right)^{2}+2 \sqrt{\varepsilon_{v \sigma}-\varepsilon_{o \sigma}} K_{o v \sigma, o^{\prime} v^{\prime} \sigma^{\prime}} \sqrt{\varepsilon_{v^{\prime} \sigma^{\prime}}-\varepsilon_{o^{\prime} \sigma^{\prime}}}\right] F_{o^{\prime} v^{\prime} \sigma^{\prime}}^{I}=\Omega_{I}^{2} F_{o v \sigma}^{I}
$$

with the coefficients

$$
C_{o v \sigma}^{I}=\sqrt{\frac{\varepsilon_{v \sigma}-\varepsilon_{o \sigma}}{\Omega_{I}}} F_{o v \sigma}^{I}
$$

In the Tamm-Dancoff approximation $\frac{1920}{20}$ excitation energies and coefficients are calculated from a different eigenvalue problem that results from eqn. (??) by setting $\mathbf{B}=0$ :

$$
\sum_{o^{\prime} \in o c c} \sum_{v^{\prime} \in v i r t} \sum_{\sigma^{\prime}} A_{o v \sigma, o^{\prime} v^{\prime} \sigma^{\prime}} C_{o^{\prime} v^{\prime} \sigma^{\prime}}^{I}=\Omega_{I} C_{o v \sigma}^{I}
$$

Using the Tamm-Dancoff (TDA) approximation in conjunction with a long-range correction (which will be introduced later) can sometimes be advantageous for two reasons:

- Firstly, it has been shown ${ }^{21 \mid 22}$ that TDA excitation energies can actually be better than those obtained from the full solution of the LR-TD-DFT equation ??, in particular, when singlet- 
triplet instabilities would lead to imaginary excitation energies.

- Secondly, when $(\mathbf{A}-\mathbf{B})$ is not diagonal, the full solution requires the computation of the matrix square root $(\mathbf{A}-\mathbf{B})^{1 / 2}$ which is computationally demanding unless one resorts to an iterative algorithm ${ }^{23}$ specifically designed to deal with eqn. (??).

The tight-binding approximation consists in replacing transition densities $\phi_{o}(\vec{r}) \phi_{v}(\vec{r})$ by transition charges $q_{A}^{o v}$ (defined later), so that the coupling matrix simplifies to

$$
K_{o v, o^{\prime} v^{\prime}} \approx \sum_{A=1}^{N_{a t}} \sum_{B=1}^{N_{a t}} q_{A}^{o v} \gamma_{A B} q_{B}^{o^{\prime} v^{\prime}} .
$$

Similarly, the transition dipoles between Kohn-Sham orbitals are reduced to sums over transition charges on different atoms:

$$
\langle o|\vec{r}| v\rangle \approx \sum_{A} \vec{R}_{A} q_{A}^{o v}
$$

Note how in going from eqn. (??) to the eqn. (??) and in approximating the coupling matrix in eqn. (??) the exchange-correlation term has been neglected for charge distributions on different atoms, arguing that the xc-functional is local. This is where the long-range correction will be put to work. Eqn. (??) is usually a reasonable approximation, unless orbitals $o$ and $v$ are located on the same atom.

\subsection{Long-range correction for TD-DFTB}

The Coulomb potential is separated into a long-range and a short-range part, ${ }^{24125}$ where the position of the smooth transition between the two regimes is controlled by a parameter $R_{\mathrm{lr}}$ :

$$
\frac{1}{r}=\underbrace{\frac{1-\operatorname{erf}\left(\frac{r}{R_{\mathrm{lr}}}\right)}{r}}_{\text {short range }}+\underbrace{\frac{\operatorname{erf}\left(\frac{r}{R_{\mathrm{lr}}}\right)}{r}}_{\text {long range }}
$$

The short range part of the exchange energy is treated with DFTB while for the long range part the exact Hartree-Fock exchange is used. Since in DFTB a local exchange correlation functional 
is employed, the short range term is essentially neglected.

The electron integrals of the screened Coulomb potential (for real-valued orbitals)

$$
(i j \mid a b)_{\mathrm{lr}}=\iint \phi_{i}\left(\vec{r}_{1}\right) \phi_{j}\left(\vec{r}_{1}\right) \frac{\operatorname{erf}\left(\frac{r_{12}}{R_{\mathrm{lr}}}\right)}{r_{12}} \phi_{a}\left(\vec{r}_{2}\right) \phi_{b}\left(\vec{r}_{2}\right) d^{3} r_{1} d^{3} r_{2}
$$

are approximated as in DFTB: ${ }^{[3}$ The transition densities between different orbitals $p^{k l}(\vec{r})=\phi_{k}(\vec{r}) \phi_{l}(\vec{r})$ are decomposed into atom-centered contributions:

$$
p^{k l}(\vec{r})=\sum_{A}^{N_{\mathrm{at}}} p_{A}^{k l}(\vec{r})
$$

Next, the monopole approximation is made assuming that the transition density due to atom $A$ is spherically symmetric around that center:

$$
p_{A}^{k l}(\vec{r})=q_{A}^{k l} F_{A}\left(\left|\vec{r}-\vec{R}_{A}\right|\right)
$$

In fact, the exact form of the functions $F_{A}(r)$ is not know. ${ }^{3}$ They can be assumed to be Gaus$\operatorname{sian}^{3}$ or Slater functions, $\frac{12}{12}$ but in either case the width of the density profile should be inversely proportional to the chemical hardness (the Hubbard parameter $U$ ) of the atom.

With these approximations the long-range two-center integrals can be written in terms of transition charges $q_{A}^{i j}$ and $q_{B}^{a b}$ :

$$
(i j \mid a b)_{\mathrm{lr}}=\sum_{A} \sum_{B} q_{A}^{i j} q_{B}^{a b} \iint F_{A}\left(\left|\vec{r}_{1}-\vec{R}_{A}\right|\right) \frac{\operatorname{erf}\left(\frac{r_{12}}{R_{\mathrm{lr}}}\right)}{r_{12}} F_{B}\left(\left|\vec{r}_{2}-\vec{R}_{B}\right|\right) d^{3} r_{1} d^{3} r_{2}
$$

The transition charges are defined as:

$$
q_{A}^{i j}=\frac{1}{2} \sum_{\mu \in A} \sum_{v}\left(c_{\mu}^{i} c_{v}^{j}+c_{\nu}^{i} c_{\mu}^{j}\right) S_{\mu v}
$$

where $S_{\mu \nu}$ is the overlap matrix between the atomic orbitals $\mu$ and $v$ and $c_{\mu}^{i}$ is the coefficient of 
the atomic orbital $\mu$ in the molecular orbital $i$.

Assuming a Gaussian- or a Slater-function form for $F_{A}(r)$ the integral for the unscreened Coulomb potential can be performed analytically ${ }^{3}$ and the result is defined as the $\gamma$-matrix:

$$
\gamma_{A B}=\iint \frac{F_{A}\left(\left|\vec{r}_{1}-\vec{R}_{A}\right|\right) F_{B}\left(\left|\vec{r}_{2}-\vec{R}_{B}\right|\right)}{\left|\vec{r}_{1}-\vec{r}_{2}\right|} d^{3} r_{1} d^{3} r_{2}
$$

For the long range part of the Coulomb potential the error-function can be taken out of the integral in eqn. ?? in a good approximation, giving the long-range $\gamma$-matrix

$$
\gamma_{A B}^{\mathrm{lr}}\left(R_{A B}\right) \approx \operatorname{erf}\left(\frac{R_{A B}}{R_{\mathrm{lr}}}\right) \gamma_{A B}\left(R_{A B}\right)
$$

from which the electron integrals with the long range part of the Coulomb potential can be calculated as:

$$
(\mu \lambda \mid \sigma v)_{\operatorname{lr}} \approx \sum_{A} \sum_{B} \gamma_{A B}^{\mathrm{lr}} q_{A}^{\mu \lambda} q_{B}^{\sigma v}
$$

Incidentally, the authors of ${ }^{12}$ have shown that the integral ?? can be transformed into a onedimensional integral that can be solved numerically (assuming the $F_{A}(r)$ 's are Slater functions). As can be seen in Fig. 1 the approximate $\gamma_{A B}^{\mathrm{lr}}$ and the numerically exact solution differ in the limit $R_{A B} \rightarrow 0$ but coincide for larger distances.

For atomic orbitals the transition densities are simply:

$$
q_{A}^{\mu \lambda}=\frac{1}{2}(\delta(\mu \in A)+\delta(\lambda \in A)) S_{\mu \lambda}
$$

Here $\delta(\mu \in A)$ is equal to 1 if the atomic orbital $\mu$ is centered on the atom $A$ and 0 otherwise. 


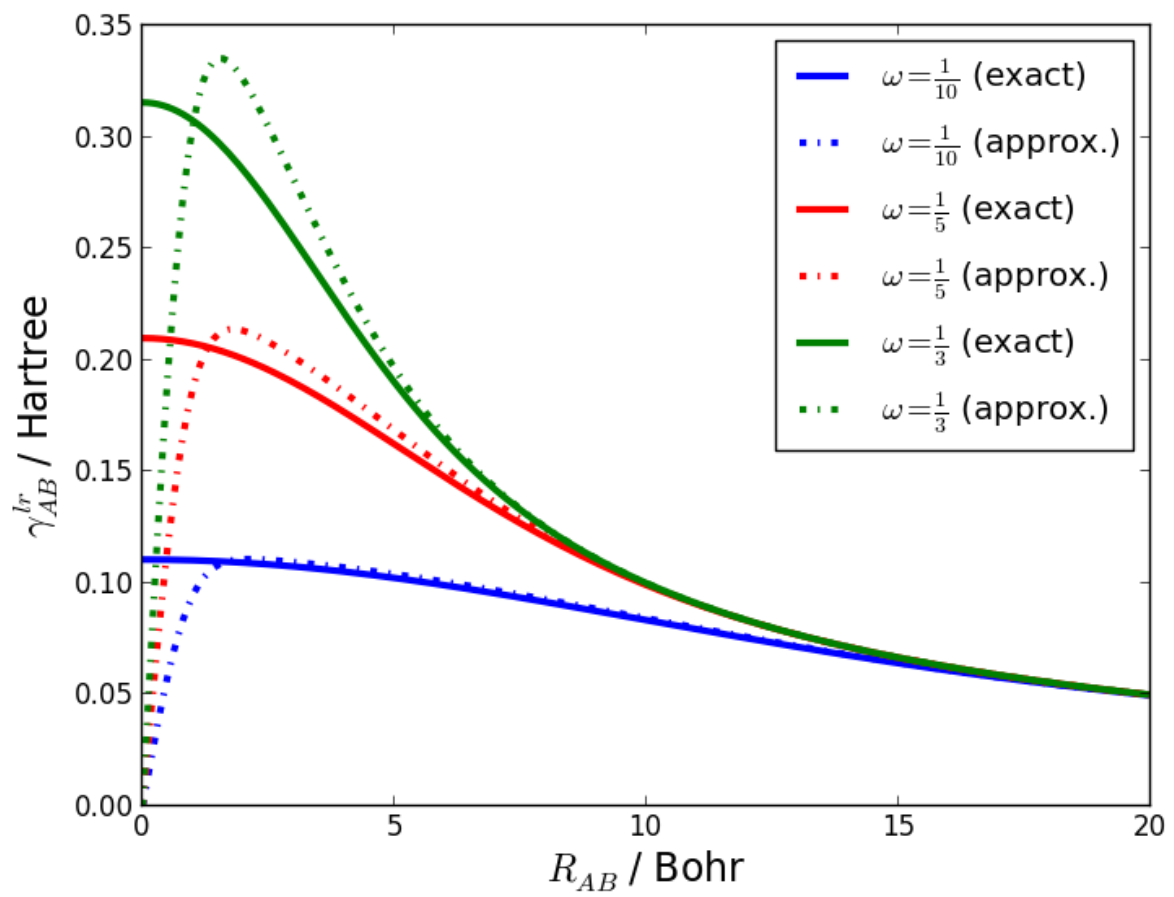

Figure 1: Comparison between the exact solution of the integral in eqn. ?? and the approximation made in eqn. ?? for different $\omega=\frac{1}{R_{l r}}$. The green curves are plotted for the long-range radius $R_{l r}=3.0$ bohr that is actually used in our parametrization. The approximation is quite accurate for interatomic distances that are relevant for charge transfer. For short atomic distances our approximation slightly overestimates $\gamma_{A B}^{l r}$ and vanishes for $R_{A B} \rightarrow 0$. In particular the diagonal elements of the Hamiltonian $H_{\mu \nu}$ are not modified, since $R_{A B}=0$ if the orbitals $\mu$ and $v$ are located on the same atom.

The long-range electron integrals in the basis of atomic orbitals can now be approximated as:

$$
\begin{aligned}
(\mu \lambda \mid \sigma v)_{\mathrm{lr}} \approx & \sum_{A} \sum_{B} \gamma_{A B}^{\mathrm{lr}} q_{A}^{\mu \lambda} q_{B}^{\sigma v}=S_{\mu \lambda} S_{v \sigma} \sum_{A} \sum_{B} \frac{1}{4} \gamma_{A B}^{\mathrm{lr}}(\delta(\mu \in A)+\delta(\lambda \in A))(\delta(\sigma \in B)+\delta(\nu \in B)) \\
= & \frac{1}{4} S_{\mu \lambda} S_{v \sigma} \sum_{A} \sum_{B} \gamma_{A B}^{\mathrm{lr}}\{\delta(\mu \in A) \delta(\sigma \in B)+\delta(\mu \in A) \delta(\nu \in B) \\
\quad+\delta(\lambda \in A) \delta(\sigma \in B)+\delta(\lambda \in A) \delta(\nu \in B)\} & \\
= & \frac{1}{4} S_{\mu \lambda} S_{v \sigma}\left\{\Gamma_{\mu \sigma}^{\mathrm{lr}}+\Gamma_{\mu \nu}^{\mathrm{lr}}+\Gamma_{\lambda \sigma}^{\mathrm{lr}}+\Gamma_{\lambda v}^{\mathrm{lr}}\right\}
\end{aligned}
$$

where the abbreviation $\Gamma_{\mu \sigma}^{\mathrm{lr}}=\sum_{A} \sum_{B} \gamma_{A B}^{\mathrm{lr}} \delta(\mu \in A) \delta(\sigma \in B)$ has been introduced.

The additional contribution to the total energy $E_{\mathrm{elec}}=E_{\mathrm{DFTB}}+E_{x}^{\mathrm{lr}}$ due to the long-range ex- 
change is:

$$
E_{x}^{\mathrm{lr}}=-\frac{1}{4} \sum_{\mu, \lambda, \sigma, v} P_{\mu \sigma} P_{\lambda v}(\mu \lambda \mid \sigma v)_{\mathrm{lr}}
$$

where $\mu, \lambda, \sigma$ and $v$ enumerate atomic orbitals, and the density matrix elements $P_{\mu \sigma}$ are computed from the molecular orbital coefficients $C_{\mu, i}$ as $P_{\mu, \sigma}=2 \sum_{i \in \mathrm{occ}} C_{\mu i} C_{\sigma i}^{*}$.

Minimization of the total energy with respect to the density matrix leads to the Kohn-Sham Hamiltonian

$$
H_{\mu \nu}^{\mathrm{KS}}=H_{\mu \nu}^{\mathrm{DFTB}}+H_{\mu \nu}^{x, \mathrm{lr}}
$$

with an additional term:

$$
\begin{aligned}
H_{\mu \nu}^{x, \mathrm{lr}}=- & \frac{1}{2} \sum_{\lambda \sigma} P_{\lambda \sigma}(\mu \lambda \mid \sigma v)_{\mathrm{lr}} \\
\stackrel{(?)}{=}- & \frac{1}{8} \sum_{\lambda \sigma} P_{\lambda \sigma} S_{\mu \lambda} S_{v \sigma}\left\{\Gamma_{\mu \sigma}^{\mathrm{lr}}+\Gamma_{\mu \nu}^{\mathrm{lr}}+\Gamma_{\lambda \sigma}^{\mathrm{lr}}+\Gamma_{\lambda v}^{\mathrm{lr}}\right\} \\
=- & \frac{1}{8}\left\{\sum_{\sigma}\left(\Gamma_{\mu \sigma}^{\mathrm{rr}}\left(\sum_{\lambda} S_{\mu \lambda} P_{\lambda \sigma}\right)\right) S_{\sigma v}\right. \\
& +\Gamma_{\mu \nu}^{\mathrm{lr}} \sum_{\sigma} \sum_{\lambda}\left(S_{\mu \lambda} P_{\lambda \sigma}\right) S_{\sigma v} \\
& +\sum_{\sigma} \sum_{\lambda}\left(S_{\mu \lambda}\left(P_{\lambda \sigma} \Gamma_{\lambda \sigma}^{\mathrm{lr}}\right)\right) S_{\sigma v} \\
& \left.+\sum_{\lambda} S_{\mu \lambda}\left(\left(\sum_{\sigma} P_{\lambda \sigma} S_{\sigma v}\right) \Gamma_{\lambda v}^{\mathrm{lr}}\right)\right\}
\end{aligned}
$$

It is important to perform the sums over the indices in such an order that they can be implemented efficiently by nested matrix multiplications.

The long-range contribution to the exchange energy in eqn. ?? can be computed as:

$$
E_{x}^{\mathrm{lr}}=-\frac{1}{8}\left\{\sum_{\mu, \sigma}\left(\sum_{\lambda} S_{\mu \lambda}\left(\sum_{v} P_{\lambda \nu} S_{\nu \sigma}\right)\right) P_{\mu \sigma} \Gamma_{\mu \sigma}^{\mathrm{lr}}+\sum_{\mu, \sigma}\left(\sum_{\lambda} S_{\mu \lambda} P_{\lambda \sigma}\right)\left(\sum_{\nu} P_{\mu \nu} S_{v \sigma}\right) \Gamma_{\mu \sigma}^{\mathrm{lr}}\right\}
$$

In the linear response formulation of TD-DFT the long-range correction leads to an additional 
term in the coupling matrix, which shifts the excitation energies of the charge transfer states up. The corrections to the A- and $\mathbf{B}$-matrices read (after separating the problem into separate singlet and triplet cases 26 :

$$
\left.\begin{array}{l}
S_{A_{o v, o^{\prime} v^{\prime}}}=\delta_{o, o^{\prime}} \delta_{v, v^{\prime}}\left(\varepsilon_{v}-\varepsilon_{o}\right)+2 K_{o v, o^{\prime} v^{\prime}}+K_{o v, o^{\prime} v^{\prime}}^{\mathrm{lr}} \\
S_{B_{o v, o^{\prime} v^{\prime}}}=2 K_{o v, v^{\prime} o^{\prime}}+K_{o v, v^{\prime} o^{\prime}}^{\mathrm{lr}}
\end{array}\right\} \text { for singlets }
$$

and

$$
\left.\begin{array}{l}
{ }^{T} A_{o v, o^{\prime} v^{\prime}}=\delta_{o, o^{\prime}} \delta_{v, v^{\prime}}\left(\varepsilon_{v}-\varepsilon_{o}\right)+K_{o v, o^{\prime} v^{\prime}}^{\mathrm{lr}} \\
{ }^{T} B_{o v, o^{\prime} v^{\prime}}=K_{o v, v^{\prime} o^{\prime}}^{\mathrm{lr}}
\end{array}\right\} \text { for triplets, }
$$

where the additional long-range coupling is given by:

$$
\begin{aligned}
& K_{o v, o^{\prime} v^{\prime}}^{\mathrm{lr}}=-\left(o o^{\prime} \mid v v^{\prime}\right)_{\mathrm{lr}} \approx-\sum_{A} \sum_{B} q_{A}^{o o^{\prime}} \gamma_{A B}^{\mathrm{lr}}\left(R_{A B}\right) q_{B}^{v v^{\prime}} \\
& K_{o v, v^{\prime} o^{\prime}}^{\mathrm{lr}}=-\left(o v^{\prime} \mid v o^{\prime}\right)_{\mathrm{lr}} \approx-\sum_{A} \sum_{B} q_{A}^{o v^{\prime}} \gamma_{A B}^{\mathrm{lr}}\left(R_{A B}\right) q_{B}^{o^{\prime} v} .
\end{aligned}
$$

$o, o^{\prime}$ are occupied and $v, v^{\prime}$ are unoccupied molecular orbitals. In addition to the transition charges between occupied and unoccupied orbitals, $q_{A}^{o v}$, which are needed for constructing the coupling matrix anyway, one has to calculate transition charges between occupied-occupied orbitals, $q_{A}^{o o^{\prime}}$, and between virtual-virtual orbitals $q_{B}^{v v^{\prime}}$.

In this approximation the quality of triplet excitation energies is expected to be much lower, as they are essentially equal to differences between Kohn-Sham orbital energies. Triplet states will be left aside in this work, since spin-unrestricted $\mathrm{DFTB}^{27}$ is necessary to describe them quantitatively and since they are dark in absorption spectra.

The oscillator strengths of singlet states are obtained as

$$
f^{I}=\frac{4}{3}\left|\sum_{o \in o c c} \sum_{v \in v i r t}\langle o|\vec{r}| v\rangle \sqrt{\Omega_{I}} C_{o v}^{I}\right|^{2} .
$$




\subsection{Slater-Koster rules for dipole matrix elements}

Transition dipole matrix elements determine the oscillator strengths in TD-DFT calculations of excited states. In DFTB they are usually approximated by transition charges located on the individual atoms,

$$
\left\langle\psi^{i}|\vec{r}| \psi^{j}\right\rangle=\sum_{\alpha} \vec{R}_{\alpha} q_{\alpha}^{i j}
$$

where $\vec{R}_{\alpha}$ is the position vector of atom $\alpha$,

$$
\begin{aligned}
\left|\psi^{i}\right\rangle & =\sum_{\mu} c_{\mu}^{i}\left|\mu\left(\vec{r}-\vec{R}_{\mu}\right)\right\rangle \\
\left|\psi^{j}\right\rangle & =\sum_{\nu} c_{\nu}^{j}\left|v\left(\vec{r}-\vec{R}_{V}\right)\right\rangle
\end{aligned}
$$

are Kohn-Sham molecular orbitals in the basis of atom-centered numerical orbitals, and the transition charges are defined as

$$
q_{\alpha}^{i j}=\frac{1}{2} \sum_{\mu \in \alpha} \sum_{\nu}\left(c_{\mu}^{i} c_{v}^{j} S_{\mu \nu}+c_{\nu}^{i} c_{\mu}^{j} S_{v \mu}\right)
$$

This approximation fails if a transition happens between molecular orbitals on the same atom, which are orthogonal by construction. In this case the overlap matrix simplifies to the identity matrix and the Mulliken approximation leads to vanishing transition charges. Therefore the excitation energies for localized $n \rightarrow \pi^{*}$ excitations are not shifted by the TD-DFT coupling matrix and no improvement of these energies relative to the Kohn-Sham energies is achieved. ${ }^{27}$ Moreover, the oscillator strengths, which determine the shape of the spectrum, can sometimes be sensitive to the way transition dipole matrix elements are computed. For example, in reference ${ }^{27}$ an on-site correction to the dipole matrix element is introduced, so that the ${ }^{2} \Pi$ states of nitric oxide, which would be dark using the Mulliken approximation, gain a small oscillator strength.

However, the oscillator strengths can also be calculated without approximation from the transition dipoles between atomic orbitals which are assembled using the Slater-Koster rules. The 
technical details are explained in the appendix C (apparently this approach has already been implemented in other DFTB codes, but is not well documented in the literature).

For transitions comprising orbitals on different atoms the oscillator strengths derived from the Mulliken charges and from the tabulated dipole matrix elements are very similar. However, when the transition is confined to a single atom, the Mulliken approximation can miss states which have weak oscillator strengths. As an example, consider the acrolein molecule, where the $S_{1}$ state is characterized by an excitation from the $n$ to the $\pi^{*}$ orbital on the oxygen. Since the oxygen is much more electronegative than the carbon it is bound to, both the $n$ and the $\pi^{*}$ orbitals consist largely of the atomic oxygen orbitals. The oscillator strength is very small, but it is not zero. Fig, 2 compares the absorption spectra when the oscillator strengths are calculated from Mulliken charges or transition dipoles, respectively.

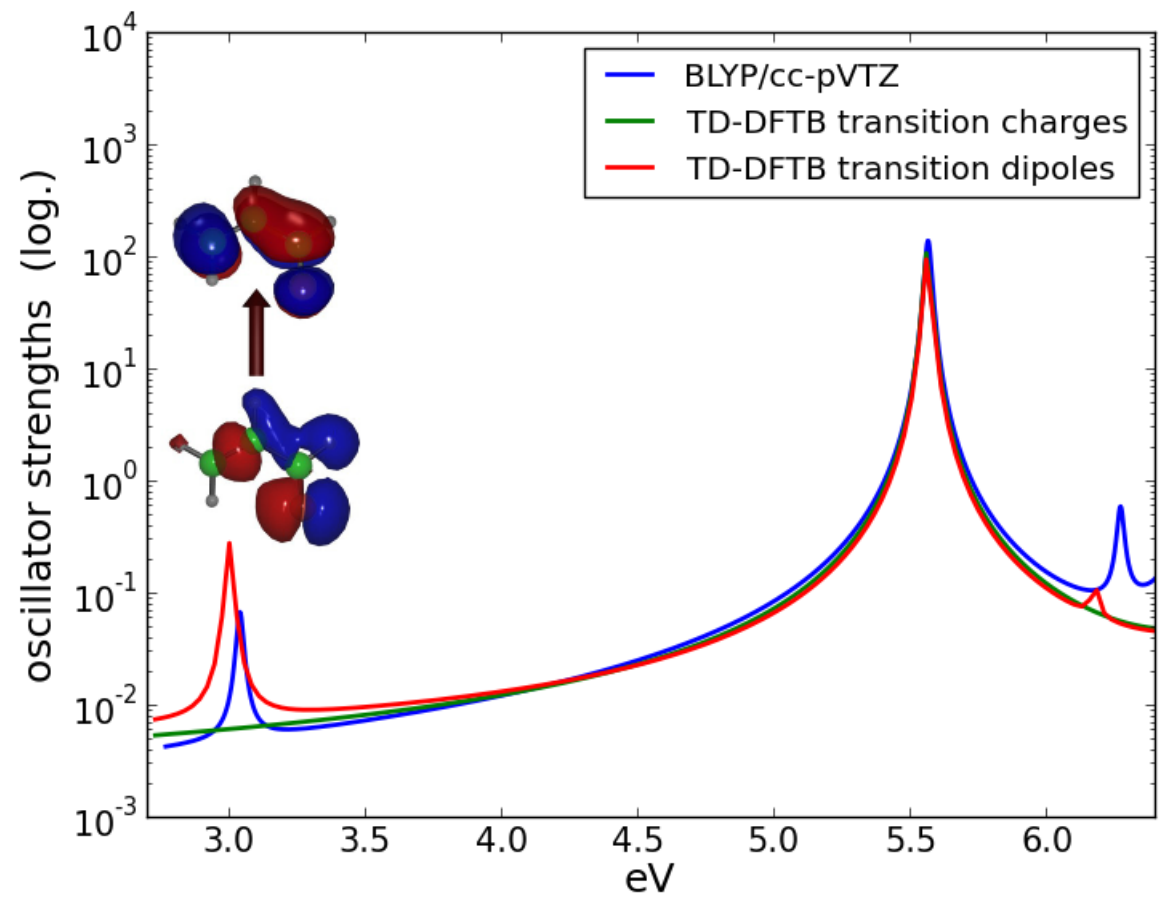

Figure 2: Absorption spectrum of acrolein. The molecular structure was optimized at the $\mathrm{BLYP}^{28[29} / \mathrm{cc}^{\mathrm{p}} \mathrm{pVTZ}^{30}$ level, and a range-separation parameter of $R_{\mathrm{lr}}=20.0 \mathrm{bohr}$ was used in the lc-TD-DFTB calculation. With the Mulliken approximation (green curve) several states disappear, although they have a small oscillator strength. For example, the lowest excited states at $\approx 3$ $\mathrm{eV}$ is of $n \rightarrow \pi^{*}$ character and has a tiny oscillator strength of $4 \cdot 10^{-4}$ which cannot be seen in the green curve. 


\subsection{Diagnosing charge transfer states}

Visual inspection of the orbitals involved in an excitation is normally required to characterize an excited state as a charge transfer (CT) state. The authors of ref. 1 introduced a simple numeric test for detecting problematic excitations. They quantified the degree of spatial overlap between occupied and virtual orbitals of an excited state $I$ using the quantity

$$
\Lambda=\sum_{o \in \mathrm{occ}} \sum_{v \in \mathrm{virt}}\left(C_{o v}^{I}\right)^{2} \int\left|\phi_{o}(\vec{r})\right|\left|\phi_{v}(\vec{r})\right| d^{3} r
$$

where $C_{o v}^{I}$ is the coefficient for the single excitation from the Kohn-Sham Slater determinant that replaces the occupied orbital $o$ by the virtual orbital $v$ in the excited state $I$.

They found that the error of DFT functionals without long-range correction such as PBE ${ }^{31}$ and B3LYP,,$\sqrt[28] 32]{ }$ correlates with the value of $\Lambda$, which can vary between 0.0 and 1.0. Values below 0.5 indicate a charge transfer or Rydberg excitation, for which PBE and B3LYP will probably be in large error.

Unfortunately, the integral over products of orbital moduli is difficult to calculate in DFTB without resorting to numerical integration. Therefore we replace the integral by

$$
O_{o v}=\int\left|\phi_{o}(\vec{r})\right|^{2}\left|\phi_{v}(\vec{r})\right|^{2} d^{3} r
$$

and define the new quantity

$$
\Lambda_{2}=\sum_{o \in \mathrm{occ}} \sum_{v \in \mathrm{virt}}\left(C_{o v}^{I}\right)^{2} \frac{O_{o v}}{\sqrt{O_{o o} O_{v v}}}
$$

that should behave similarly to $\Lambda$ from eqn. ??. Applying again the monopole approximation, $O_{o v}$ can be approximated by

$$
O_{o v} \approx \sum_{A} \sum_{B} q_{A}^{o o} q_{B}^{v v} \underbrace{\int F_{A}\left(\left|\vec{r}-\vec{R}_{A}\right|\right) F_{B}\left(\left|\vec{r}-\vec{R}_{B}\right|\right) d^{3} r}_{\Omega_{A B}}
$$


The overlap integral $\Omega_{A B}$ of the spherical charge distributions centered on atoms $A$ and $B$ can be performed analytically assuming that $F$ has a Gaussian profile:

$$
F_{A}(\vec{r})=\frac{1}{\left(2 \pi \sigma_{A}^{2}\right)^{3 / 2}} \exp \left(-\frac{|\vec{r}|^{2}}{2 \sigma_{A}^{2}}\right)
$$

where the width of the distribution is inversely proportional to the Hubbard parameter of atom A (see eqn. 29 of ref. $\stackrel{3}{\text { ) }}$ :

$$
\sigma_{A}=\frac{1}{\sqrt{\pi}} \frac{1}{U_{A}}
$$

The integral is:

$$
\Omega_{A B}=\frac{1}{\left(2 \pi\left(\sigma_{A}^{2}+\sigma_{B}^{2}\right)\right)^{3 / 2}} \exp \left(-\frac{1}{2} \frac{1}{\sigma_{A}^{2}+\sigma_{B}^{2}}\left(\vec{R}_{A}-\vec{R}_{B}\right)^{2}\right)
$$

For extended molecular systems the analysis of charge transfer by looking at the orbitals can become very cumbersome as the grids on which they are stored have to be very large. In the appendix $\mathrm{F}$ we provide an alternative analysis method in terms of the difference density between ground and excited stated, which is partitioned into atomic contributions.

\section{Computational Details}

The values for the confinement radius $r_{0}$ and the Hubbard parameter $U_{H}$ that were used to parametrize the electronic part of DFTB are shown in Table 1. The Kohn-Sham equations with the PW92 33 local exchange-correlation functional for the pseudo atoms were solved self-consistently using the shooting method. ${ }^{[34}$ In order to quickly assemble the DFTB Hamiltonian and the transition dipoles, Slater-Koster tables were generated for all pairwise combinations of valence orbitals for the atoms $\mathrm{H}, \mathrm{C}, \mathrm{N}$ and $\mathrm{O}$. The parameter for the long range correction was set to $R_{\mathrm{lr}}=3.03 \mathrm{bohr}$ (for comparison, CAM-B3LYP ${ }^{13}$ uses $R_{\mathrm{lr}}=\frac{1}{\mu}=3.03 \mathrm{bohr}$, but only $65 \%$ exact exchange). So far we did not try to find an optimum value for $R_{\mathbf{I r}}$.

The structures of the test molecules were taken from the database ${ }^{38}$ that has been published 
Table 1: Electronic DFTB parameters. In general, the Hubbard parameter is set to the difference between experimental ionization energy and electron affinity, $U_{H}=\mathrm{IE}^{\sqrt{35}}$-EA, ${ }^{36}$ where available, and the confinement radius is taken as $1.85 \times$ the covalent radius as reported in, ${ }^{37} r_{0}=1.85 r_{\text {cov }}$. Deviating from this rule, the Hubbard parameter of nitrogen was set to 0.530 Hartree (taken from the DFTB implementation of Hotbit ${ }^{3}$ ), since there is no experimental value for the electron affinity available (the $N^{-}$anion is unstable).

\begin{tabular}{r|r|r} 
Atom & $r_{0} /$ bohr & $U_{H} /$ Hartree \\
\hline \hline $\mathrm{H}$ & 1.084 & 0.472 \\
$\mathrm{C}$ & 2.657 & 0.367 \\
$\mathrm{~N}$ & 2.482 & 0.530 \\
$\mathrm{O}$ & 2.307 & 0.447
\end{tabular}

by the authors of Ref. ${ }^{1}$ The molecules $\mathrm{N}_{2}, \mathrm{CO}, \mathrm{H}_{2} \mathrm{CO}$ and $\mathrm{HCl}$ were excluded because the minimal basis set of occupied valence orbitals used in DFTB is not suitable for describing Rydberg states. In the calculations labeled as lc-TD-DFTB the long-range correction as described above was included, Casida's equation ?? was used for solving the linear response equations and the transition dipoles were assembled from Slater-Koster files. In the calculations labeled as TD-DFTB the long-range correction was absent and the transition dipoles were calculated using transition charges. For comparison we also determined the excitation energies and oscillator strengths with full TD-DFT using the CAM-B3LYP ${ }^{13}$ functional and the cc-pVTZ ${ }^{30}$ basis set as implemented in the Gaussian $09^{39}$ program package.

\section{Results}

Energies and oscillator strengths of specific excitations computed with TD-DFTB, lc-TD-DFTB and CAM-B3LYP are compared in Table 2. The HOMO-LUMO gaps are plotted in Fig. 4.

Peptides. The first molecules in the test set are three model peptides of increasing length, a dipeptide, a $\beta$-dipeptide and a tripeptide. The labels $n\left(O_{i}\right), \pi\left(N_{i}\right)$ and $\pi_{i}^{*}$, which are used to classify the excited states, refer to the lone electron pair on the the $i$-th oxygen, the binding $\pi$ orbital containing the $i$-th nitrogen and the anti-bonding $\pi$-orbital on the $i$-th carbonyl group. The ordering of the carbonyl groups and atoms is depicted in Fig. 3 . 
The energies of the lowest localized excitations are well reproduced both by TD-DFTB and lcTD-DFTB. The energies of charge transfer excitations are underestimated, although lc-TD-DFTB puts them much closer to the CAM-B3LYP results and correctly predicts that the band of charge transfer states should be located above the local excitations. As opposed to this, TD-DFTB produces charge transfer states that lie below the lowest local excitation. This problem gets worse as the peptide grows: In the tripeptide TD-DFTB erroneously predicts the lowest excitation to be a long-range charge transfer from $n\left(O_{1}\right)$ to $\pi_{3}^{*}$ with an energy of $4.59 \mathrm{eV}$, whereas the CAM-B3LYP energy of this excitation would be $8.68 \mathrm{eV}$.

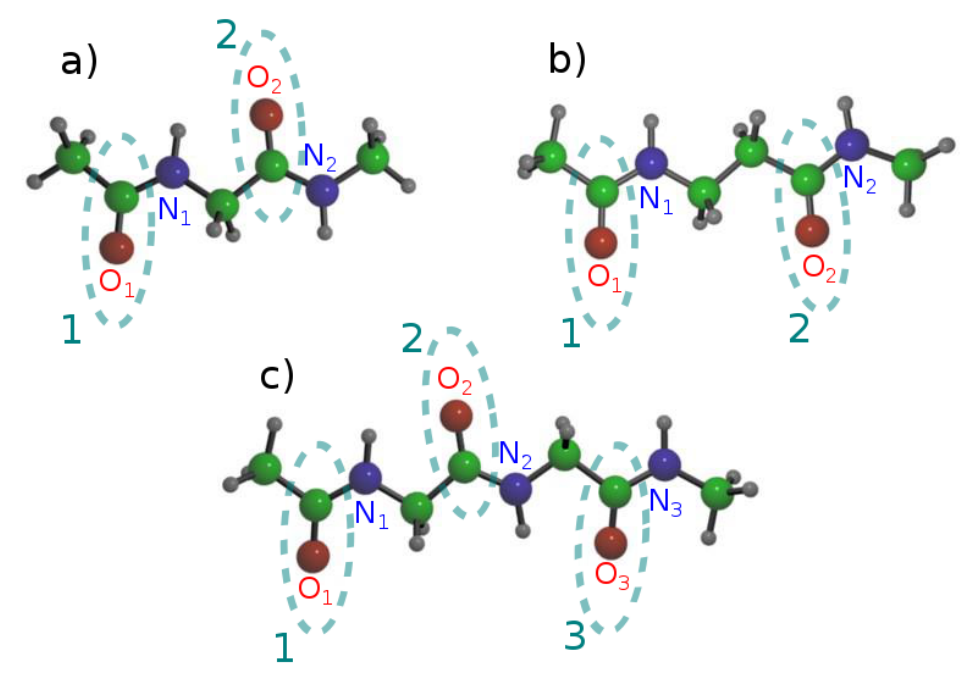

Figure 3: Peptide structures. a) dipeptide, b) $\beta$-dipeptide and c) tripeptide. The carbonyl groups used to classify the excitations are encircled.

Acenes. The next molecules are the smallest 4 polyacenes Naphthalene $(n=1)$, Anthracene $(n=2)$, Tetracene $(n=3)$ and Pentacene $(n=4)$. The large degree of conjugation leads to orbitals that are delocalized over the entire molecule. TD-DFTB underestimates the energies of all states consistently by $<0.5 \mathrm{eV}$, and this error remains stable with the size of the acenes. With lc-TDDFTB the $B_{2 u}$ states deviate no more than $0.1 \mathrm{eV}$ from the CAM-B3LYP reference values.

N-phenylpyrrole. In this heterocyclic aromatic compound, TD-DFTB underestimates local excitations by $\approx 1 \mathrm{eV}$ while lc-TD-DFTB is correct to within $0.1 \mathrm{eV}$. Without long-range correction the lowest state with $A_{1}$ symmetry has charge transfer character $\left(\Lambda_{2}=0.02\right)$. The long-range 
correction shifts this state to higher energies so that the lowest $A_{1}$ state now belongs to a local excitation $\left(\Lambda_{2}=0.49\right)$, as it should.

The second $B_{2}$ and $A_{1}$ states, which involve an electron transfer from the pyrrole ring to the benzene ring, are predicted far too low in energy by TD-DFTB.

DMABN. 4-(N,N-dimethylamino)benzonitrile (DMABN) possesses a low-lying charge transfer state that is formed when the nitrogen on one side of the phenyl ring donates charge to the $-\mathrm{C} \equiv \mathrm{N}$ group on the opposite side. Although the ordering of the states is correct even without long-range correction, lc-TD-DFTB comes much closer to the reference values than plain TD-DFTB.

Polyacetylenes. As with the acenes, TD-DFTB energies are too low by $0.5 \mathrm{eV}$ as compared to the lc-TD-DFTB and CAM-B3LYP values.

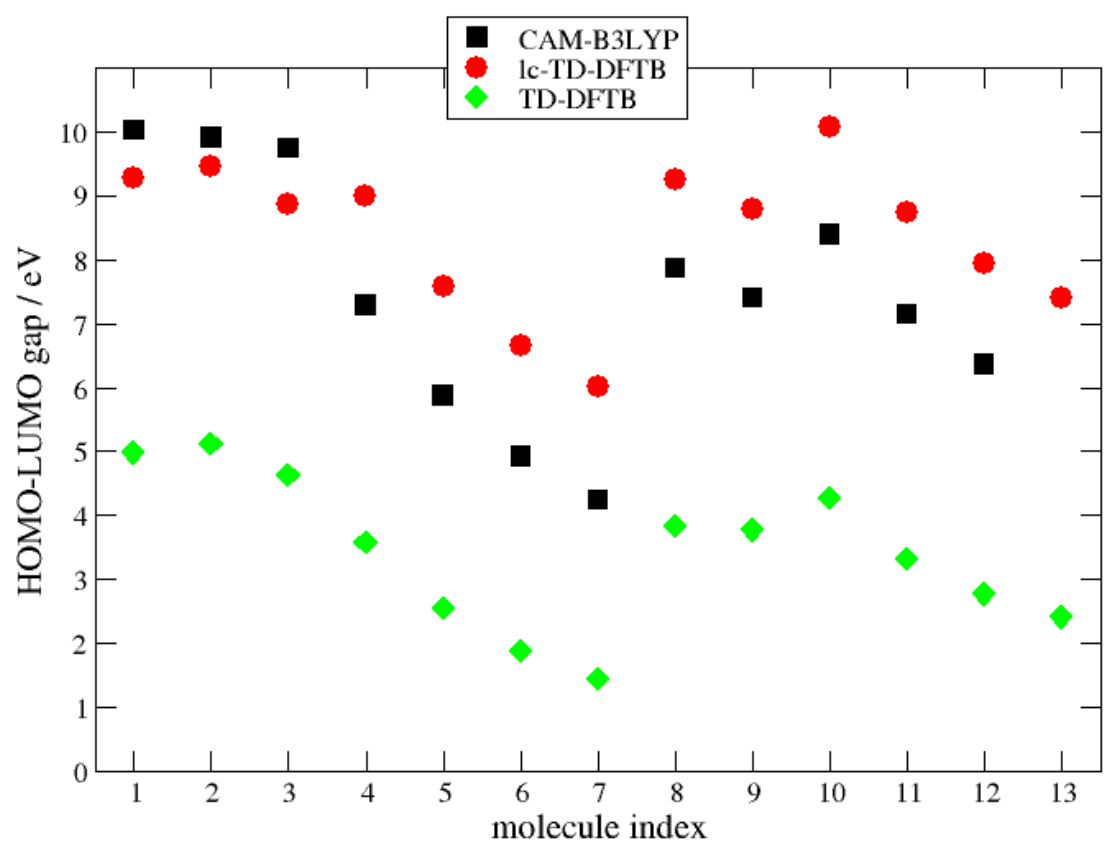

Figure 4: HOMO-LUMO gaps for the molecules from the test set. The names of the molecules encoded by the numbers on the $\mathrm{x}$-axis can be found in the first column of table 2. As expected from a long-range corrected functional the HOMO-LUMO gaps are larger for lc-TD-DFTB than for TD-DFTB. 
Table 2: TD-DFTB and lc-TD-DFTB excitation energies and reference values from TD-DFT calculations at the CAM-B3LYP/cc-pVTZ level. Energies are in $\mathrm{eV}$ and oscillator strengths are given in brackets. The type of excitation ( $\mathrm{L}=$ local, $\mathrm{CT}=$ charge transfer, $\mathrm{DL}=$ delocalized) was assigned by inspecting the dominant pair of occupied and virtual orbitals in the transitions. The $\Lambda_{2}$ values are calculated for lc-TD-DFTB.

\begin{tabular}{|c|c|c|c|c|c|c|c|}
\hline & Molecule & State & $\overline{\Lambda_{2}}$ & Type & $\overline{\text { TD-DFTB }}$ & le-TD-DFTB & CAM-B3LYP \\
\hline \multirow[t]{4}{*}{1} & Dipeptide & A" $n\left(O_{1}\right) \rightarrow \pi_{1}^{*}$ & 0.61 & $\mathrm{~L}$ & $5.48(0.000)$ & $5.33(0.000)$ & $5.68(0.001)$ \\
\hline & & A" $n\left(O_{2}\right) \rightarrow \pi_{2}^{*}$ & 0.75 & $\mathrm{~L}$ & $5.44(0.000)$ & $5.40(0.000)$ & $5.92(0.001)$ \\
\hline & & $\mathrm{A}^{\prime} \pi\left(N_{1}\right) \rightarrow \pi_{2}^{*}$ & 0.08 & CT & $5.54(0.002)$ & $6.23(0.015)$ & $7.00(0.010)$ \\
\hline & & A" $n\left(O_{1}\right) \rightarrow \pi_{2}^{*}$ & 0.20 & $\mathrm{CT}$ & $4.92(0.000)$ & $5.98(0.000)$ & $7.84(0.000)$ \\
\hline \multirow[t]{4}{*}{2} & $\beta$-dipeptide & A" $n\left(O_{1}\right) \rightarrow \pi_{1}^{*}$ & 0.77 & $\mathrm{~L}$ & $5.47(0.000)$ & $5.39(0.000)$ & $5.67(0.001)$ \\
\hline & & A" $n\left(O_{2}\right) \rightarrow \pi_{2}^{*}$ & 0.77 & $\mathrm{~L}$ & $5.45(0.000)$ & $5.39(0.000)$ & $5.76(0.000)$ \\
\hline & & $\mathrm{A}^{\prime} \pi\left(N_{1}\right) \rightarrow \pi_{2}^{*}$ & 0.56 & CT & $5.64(0.000)$ & $7.37(0.558)$ & $7.42(0.328)$ \\
\hline & & A" $n\left(O_{1}\right) \rightarrow \pi_{2}^{*}$ & 0.01 & $\mathrm{CT}$ & $5.05(0.000)$ & $6.71(0.000)$ & $8.38(0.008)$ \\
\hline \multirow[t]{9}{*}{3} & Tripeptide & A" $n\left(O_{1}\right) \rightarrow \pi_{1}^{*}$ & 0.59 & $\mathrm{~L}$ & $5.51(0.000)$ & $5.34(0.000)$ & $5.72(0.001)$ \\
\hline & & A" $n\left(O_{2}\right) \rightarrow \pi_{2}^{*}$ & 0.58 & $\mathrm{~L}$ & $5.50(0.000)$ & $5.37(0.000)$ & $5.93(0.001)$ \\
\hline & & A" $n\left(O_{3}\right) \rightarrow \pi_{3}^{*}$ & 0.72 & $\mathrm{~L}$ & $5.44(0.000)$ & $5.43(0.000)$ & $6.00(0.001)$ \\
\hline & & $\mathrm{A}^{\prime} \pi\left(N_{1}\right) \rightarrow \pi_{2}^{*}$ & 0.07 & CT & $5.56(0.002)$ & $6.25(0.014)$ & $6.98(0.014)$ \\
\hline & & $\mathrm{A}^{\prime} \pi\left(N_{2}\right) \rightarrow \pi_{3}^{*}$ & 0.16 & CT & $5.78(0.002)$ & $6.52(0.032)$ & $7.68(0.102)$ \\
\hline & & A" $n\left(O_{1}\right) \rightarrow \pi_{2}^{*}$ & 0.19 & CT & $4.93(0.000)$ & $5.99(0.000)$ & $7.78(0.000)$ \\
\hline & & A" $n\left(O_{2}\right) \rightarrow \pi_{3}^{*}$ & 0.28 & CT & $5.16(0.000)$ & $6.33(0.000)$ & $8.25(0.000)$ \\
\hline & & $\mathrm{A}^{\prime} \pi\left(N_{1}\right) \rightarrow \pi_{3}^{*}$ & 0.03 & $\mathrm{CT}$ & $5.20(0.000)$ & $8.35(0.000)$ & $8.51(0.007)$ \\
\hline & & A" $n\left(O_{1}\right) \rightarrow \pi_{3}^{*}$ & 0.01 & CT & $4.59(0.000)$ & $9.04(0.000)$ & $8.68(0.000)$ \\
\hline \multirow[t]{2}{*}{4} & Acene $(\mathrm{n}=1)$ & $B_{2 u}$ & 0.83 & DL & $4.27(0.007)$ & $4.53(0.006)$ & $4.62(0.000)$ \\
\hline & & $B_{1 u}$ & 0.77 & DL & $4.02(0.044)$ & $4.84(0.046)$ & $4.67(0.071)$ \\
\hline \multirow[t]{2}{*}{5} & Acene $(n=2)$ & $B_{1 u}$ & 0.77 & DL & $3.00(0.047)$ & $3.84(0.067)$ & $3.53(0.076)$ \\
\hline & & $B_{2 u}$ & 0.83 & DL & $3.66(0.026)$ & $4.02(0.021)$ & $4.04(0.001)$ \\
\hline \multirow[t]{2}{*}{6} & Acene $(n=3)$ & $B_{1 u}$ & 0.74 & DL & $2.32(0.040)$ & $3.19(0.073)$ & $2.76(0.071)$ \\
\hline & & $B_{2 u}$ & 0.82 & DL & $3.27(0.056)$ & $3.69(0.047)$ & $3.65(0.003)$ \\
\hline \multirow[t]{2}{*}{7} & Acene $(n=4)$ & $B_{1 u}$ & 0.72 & DL & $1.85(0.033)$ & $2.74(0.076)$ & $2.22(0.064)$ \\
\hline & & $B_{2 u}$ & 0.81 & DL & $3.01(0.100)$ & $3.48(0.087)$ & $3.39(0.008)$ \\
\hline \multirow[t]{4}{*}{8} & N-phenylpyrrole & $B_{2}$ & 0.48 & $\mathrm{~L}$ & $3.96(0.005)$ & $4.98(0.003)$ & $5.06(0.013)$ \\
\hline & & $A_{1}$ & 0.49 & $\mathrm{~L}$ & $3.99(0.000)$ & $5.07(0.458)$ & $5.12(0.365)$ \\
\hline & & $B_{2}$ & 0.29 & CT & $4.30(0.009)$ & $5.24(0.015)$ & $5.27(0.015)$ \\
\hline & & $A_{1}$ & 0.02 & CT & $4.51(0.352)$ & $6.18(0.000)$ & $5.92(0.179)$ \\
\hline \multirow[t]{2}{*}{9} & DMABN & $B$ & 0.37 & $\mathrm{~L}$ & $4.27(0.023)$ & $4.47(0.022)$ & $4.72(0.024)$ \\
\hline & & $A$ & 0.49 & CT & $4.52(0.308)$ & $4.95(0.453)$ & $4.91(0.520)$ \\
\hline 10 & Polyacetylene $(n=2)$ & $B_{u}$ & 0.68 & $\mathrm{DL}$ & $5.57(0.500)$ & $6.21(0.420)$ & $6.04(0.706)$ \\
\hline 11 & Polyacetylene $(\mathrm{n}=3)$ & $B_{u}$ & 0.69 & DL & $4.54(0.813)$ & $5.10(0.707)$ & $5.03(1.110)$ \\
\hline 12 & Polyacetylene $(n=4)$ & $B_{u}$ & 0.69 & DL & $3.88(1.133)$ & $4.43(0.995)$ & $4.39(1.533)$ \\
\hline 13 & Polyacetylene $(\mathrm{n}=5)$ & $B_{u}$ & 0.69 & DL & $3.41(1.437)$ & $3.98(1.269)$ & $3.94(1.961)$ \\
\hline
\end{tabular}




\subsection{Correlation between errors and $\Lambda_{2}$}

When the deviations of the excitation energies relative to the CAM-B3LYP values are plotted against the degree of spatial overlap $\Lambda_{2}$ (see Fig. 5), a clear correlation is visible. $\Lambda_{2}$ values close to 0.0 can be associated with charge transfer states, values around 0.5 with local excitations and values close to 1.0 with strongly delocalized excitations. For charge transfer states, the long range correction reduces the maximum error from -4.0 to $-2.0 \mathrm{eV}$. For local excitations, TD-DFTB and lc-TD-DFTB have similar errors - TD-DFTB underestimates energies by at most $0.5 \mathrm{eV}$, while lc-TD-DFTB overestimates them by the same amount. For strongly delocalized excitations, as they occur in the acene series, lc-TD-DFTB shifts the error to the positive region with respect to TD-DFTB, and reduces somewhat the absolute values of the error.

a)

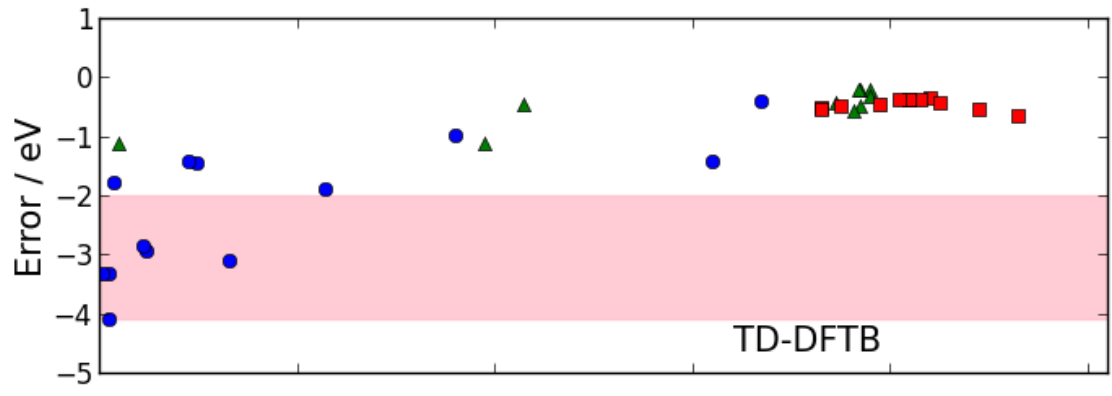

b)

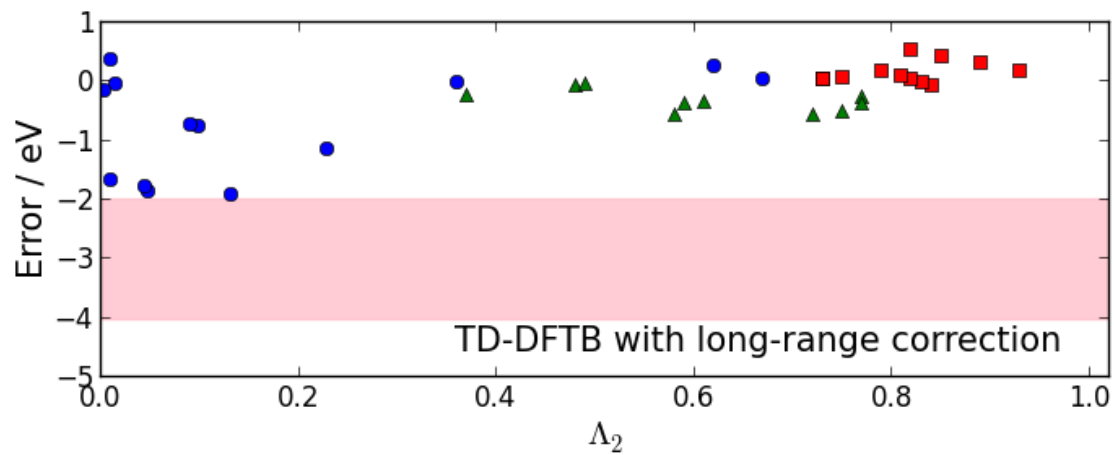

Figure 5: Deviation of excitation energies from CAM-B3LYP reference values for a) TD-DFTB and b) lc-TD-DFTB plotted against $\Lambda_{2}$ (a measure of spatial overlap defined in eqn. ??), local excitations $(\boldsymbol{\Delta})$, charge transfer excitations $(\bullet)$, delocalized excitations $(\boldsymbol{\square})$. The area into which errors from charge transfer states fall without long-range correction is highlighted in pink.

Table 3 gives the mean errors averaged over the whole set of test molecules. In summary, 
energies of localized, charge transfer as well as delocalized states are systematically improved by lc-TD-DFTB. In particular, the long range correction particularly markedly the energies of delocalized and charge transfer states while the energies of the localized states are only marginally better.

Table 3: Mean errors (in eV) relative to the CAM-B3LYP excitation energies for the molecules in the test set. The long-range correction particularly improves energies of delocalized and charge transfer states.

\begin{tabular}{ccccc} 
Method & Total & Local & Charge Transfer & Delocalized \\
\hline TD-DFTB & 1.13 & 0.51 & 2.22 & 0.46 \\
lc-TD-DFTB & 0.46 & 0.34 & 0.83 & 0.17
\end{tabular}

\subsection{Are potential energy surfaces with lc-TD-DFTB accurate enough?}

The good agreement of lc-TD-DFTB absorption spectra with those obtained from full long-range corrected TD-DFT around equilibrium geometries unfortunately does not guarantee that global features of the excited potential energy surfaces are equally well reproduced.

We have investigated the accuracy of the excited state potentials by exploring profiles of the excitation energies along the torsion angles in two of the molecules from the test set, DMABN and N-phenylpyrrole (see insets in Figs. 6 and 7 for the definition of the torsion angle $\tau$ ), and tried to identify the approximations in TD-DFTB that lead to the discrepancies with respect to full TD-DFT.

Both molecules show dual fluorescence ${ }^{40[41}$ since the excited charge transfer state has an energy minimum at a dihedral angle of $90^{\circ}$, while the ground state is more or less planar. As shown for DMABN, $\stackrel{42}{ }$ density functionals without long-range correction such as PBE or B3LYP fail to predict an energy maximum of the charge transfer state at the twisted geometry. Only long-range corrected functionals are faithful to the shape of the potential energy surface.

In order to separate the influence of the functional from the influence of the basis size, we first compare the torsion profiles for the $\mathrm{PBE}^{\frac{31}{1}}$ with those of the LC-PBE functional ${ }^{25}$ which is the long-range corrected version of PBE - both with the aug-cc-pVDZ ${ }^{30}$ basis set (Fig.6a) vs. b) and 
Fig.7 7 a) vs. b)). In the next step we reduce the basis to a minimal one (STO-3G ${ }^{43}$ ) (Fig 6 c) vs. d) and Fig.7k) vs. d)). Clearly, the absence of a long-range exchange term changes the profiles qualitatively, whereas the reduction of the basis size does not - except for a large energy increase of all excited states. Now, the stage is set for comparing with tight-binding DFT.

In standard TD-DFTB, the charge transfer states collapse to a minimum at $\tau=90^{\circ}$ for both molecules as expected of a functional lacking long-range exchange (Fig.6f) and Fig,7f)). Density functionals with local exchange cannot describe charge transfer over long distances because the transition density $\phi_{o}(\vec{r}) \phi_{v}(\vec{r})$ vanishes if the occupied and virtual orbital are spatially separated. $\underline{44}$

For lc-TD-DFTB, one would expect an improved description of the charge transfer states. While the energy maximum at the twisted geometry is at least qualitatively reproduced for $\mathrm{N}$ phenylpyrrole (Fig.7e),g)), for DMABN lc-TD-DFTB performs even worse than TD-DFTB (Fig6e)): The lc-TD-DFTB torsion profile shows an even more pronounced dip at $90^{\circ}$, but for a different reason. This becomes evident from Tables 4 and 5 which show the blocks of the long-range part of the coupling matrix $K_{l r}$ that mixes different transitions between frontier orbitals. The lowest two excited states in DMABN are dominated by excitations from the HOMO-1 or HOMO into the LUMO or LUMO+1. A small off-diagonal matrix element of -0.01 is present at $\tau=0^{\circ}$, whereas at $\tau=90^{\circ}$ the block becomes almost diagonal (the largest off-diagonal element is below smaller than 0.0001). Therefore at the twisted geometry, the coupling matrix cannot correct the excitation energies. The vanishing of the long-range coupling is probably due to the Mulliken approximation that reduces the transition density to spherically symmetric charge distributions around atoms.

Table 4: long-range coupling matrix $K_{l r}$ for the frontier orbitals at $\tau=0^{\circ}$ for DMABN.

\begin{tabular}{c|ccc}
$K_{l r}$ & $\mathrm{H}-1 \rightarrow \mathrm{L}$ & $\mathrm{H} \rightarrow \mathrm{L}$ & $\mathrm{H} \rightarrow \mathrm{L}+1$ \\
\hline $\mathrm{H}-1 \rightarrow \mathrm{L}$ & 0.1798 & 0.0000 & -0.0106 \\
$\mathrm{H} \rightarrow \mathrm{L}$ & 0.0000 & 0.1543 & -0.0000 \\
$\mathrm{H} \rightarrow \mathrm{L}+1$ & -0.0106 & -0.0000 & 0.1721
\end{tabular}

Comparing the torsion profile of N-phenylpyrrole for the aug-cc-pVDZ and the minimal STO$3 \mathrm{G}$ basis sets allows one to identify a second source of error. Diffuse orbitals probably play a large role in charge transfer states, where orbitals participating in a transition overlap only slightly with 
Table 5: long-range coupling matrix $K_{l r}$ at $\tau=90^{\circ}$ for DMABN.

\begin{tabular}{c|ccc}
$K_{l r}$ & $\mathrm{H}-1 \rightarrow \mathrm{L}$ & $\mathrm{H} \rightarrow \mathrm{L}$ & $\mathrm{H} \rightarrow \mathrm{L}+1$ \\
\hline $\mathrm{H}-1 \rightarrow \mathrm{L}$ & 0.1520 & -0.0001 & -0.0001 \\
$\mathrm{H} \rightarrow \mathrm{L}$ & -0.0001 & 0.1590 & -0.0000 \\
$\mathrm{H} \rightarrow \mathrm{L}+1$ & -0.0001 & -0.0000 & 0.1690
\end{tabular}

each other's fuzzy tails. The torsion profile at the LC-PBE/STO-3G level (Fig. 7 7 c)) is comparable to lc-TD-DFTB (Fig. 7]e) and d)), which also uses a minimal set of valence orbitals. In both cases the maxima of the lowest 3 excited states are less pronounced than in the LC-PBE/aug-cc-pVDZ (Fig. 7 a)) case. 


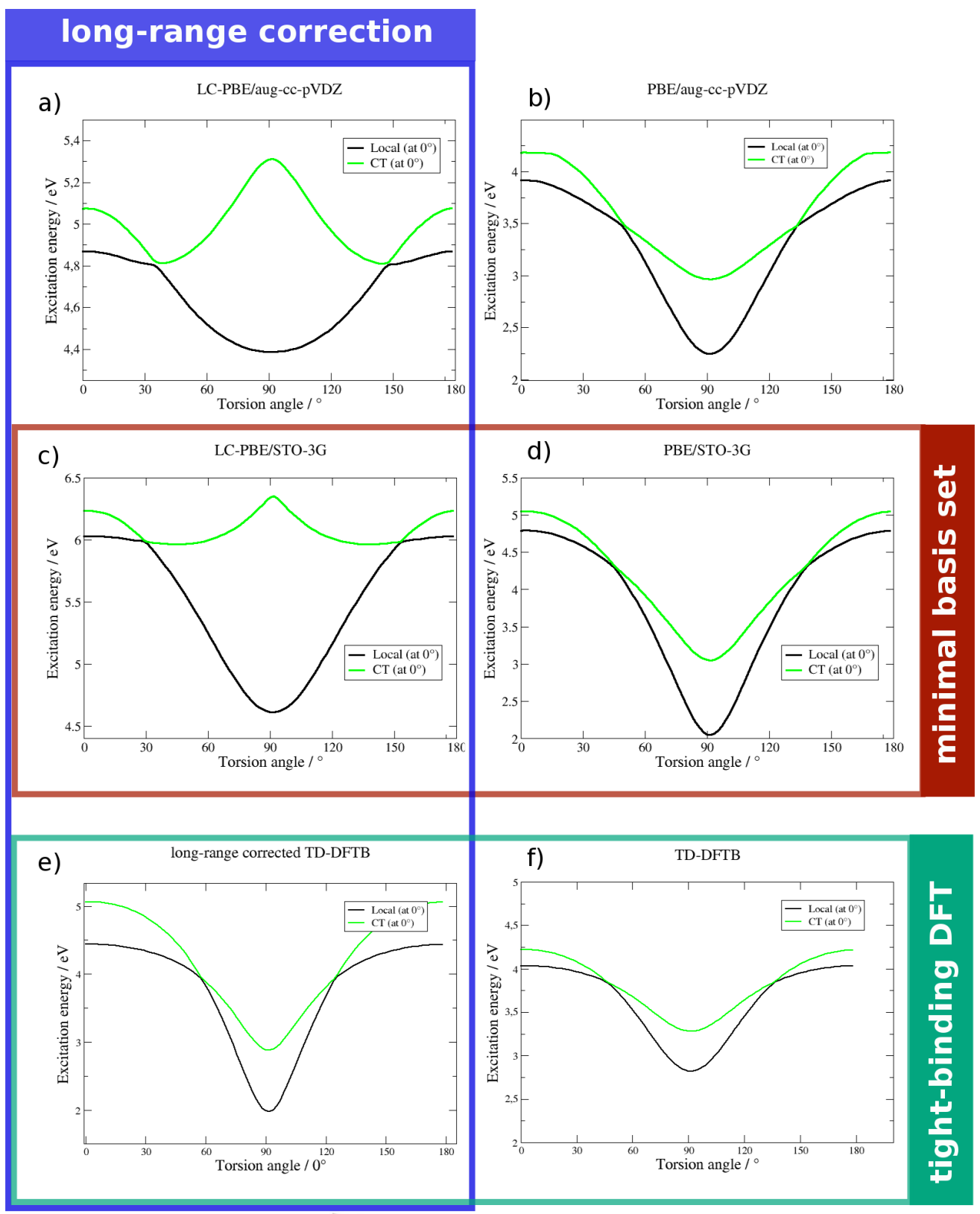

g)

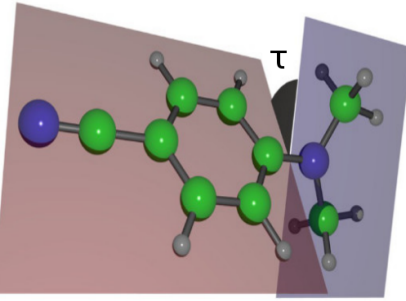

Figure 6: Excitation energy profile of DMABN along torsion angle. a),b),c) and d) with full TD-DFT using the PBE functional; e) and f) with tight-binding DFT. 


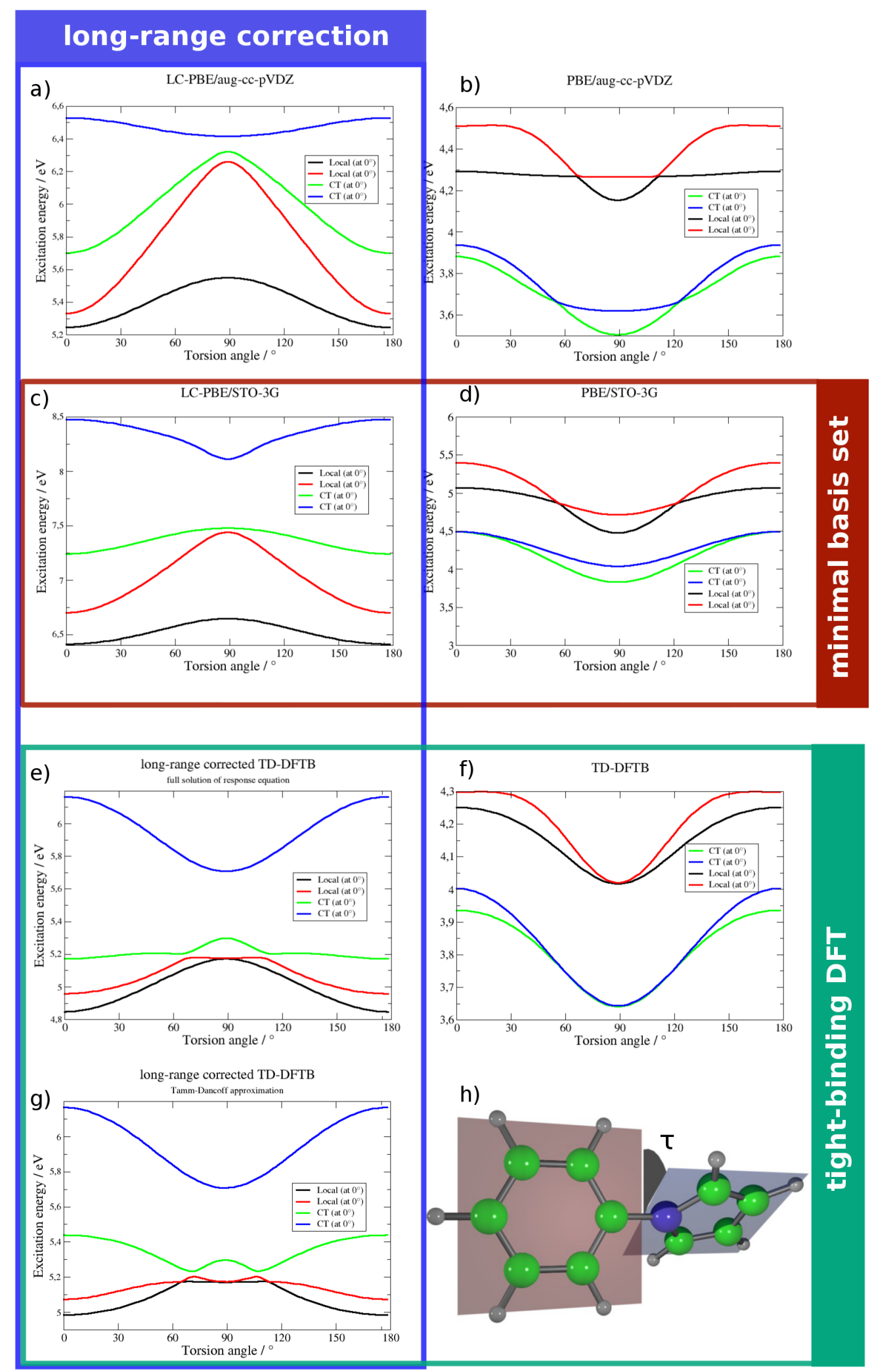

Figure 7: Excitation energy profile of N-phenylpyrrole along torsion angle. a),b),c) and d) with full TD-DFT using the PBE function; e),f) and g) with tight-binding DFT. In e) the lc-TD-DFTB spectrum has been obtained from the full solution of eqn. ?? while in $\mathbf{g}$ ) the Tamm-Dancoff approximation was employed. 


\section{Conclusion and Outlook}

Tight-binding DFT is a very efficient method for calculating the electronic structure of large organic molecules around their equilibrium structures. This efficiency rests on the precalculation of matrix elements using the Slater-Koster rules and on approximations for the two-center integrals using Mulliken charges. Any improvement of the method should not deviate from these principles as it would forfeit speed of execution. We have presented two improvements that add very little computational overhead, the long-range correction in the spirit of CAM-B3LYP and the calculation of oscillator strengths from Slater-Koster files for transition dipoles. In full TD-DFT, the computational cost increases considerably if range-separated functionals are used, not so in the tight-binding approximation.

Two measures of charge transfer were tested which can be extracted easily from TD-DFTB calculations, the quantity $\Lambda_{2}$ and the particle-hole separation. These can be used to detect cases of problematic behaviour involving long charge-transfer character of excited states. Application of the method to a test set of molecules with problematic charge transfer states led to an average error of $0.3 \mathrm{eV}$ for local excitations, $0.8 \mathrm{eV}$ for charge transfer excitations and $0.2 \mathrm{eV}$ for delocalized excitations. Especially energies of charge transfer states are improved by over $1.0 \mathrm{eV}$ relative to tight-binding DFT without range-separation. A more general test of the method for a set of common organic molecules ${ }^{45}$ is compiled in the supporting information.

Of course, being a semi-empirical method, the errors of lc-TD-DFTB also depend on the right choice of transferable parameters and more work needs to focus on optimizing these parameters, in particular the range-separation radius $R_{\mathrm{lr}}$ and the choice of the switching function (see appendix B. For simplicity, we derived the electronic parameters (the confinement radius $r_{0}$ and the Hubbard parameter $U_{H}$ ) from experimental data. Previous work on the parametrization of DFTB ${ }^{17 / 46 / 47}$ has proven that, by fitting the parameters to full DFT calculations on training sets of representative molecules, the accuracy of ab-initio electronic structure calculations can be reached.

In the future, we intend to use lc-TD-DFTB to study polymers containing e. g. organic chro- 
mophores. In this context, a comparison with other semi-empirical methods is pertinent. While some approximations are similar to semi-empirical wavefunction based methods, the derivation from time-dependent density functional theory is advantageous from the point of view of computational efficiency since only single excitations are included. This approximation is often sufficient for describing low-lying bright excited states. The problem that the active space grows quickly with the system size becomes relevant only for much larger systems, when the coupling matrix cannot be diagonalized with all single excitations included. Therefore the method is suited for predicting absorption spectra of large systems with a few thousand second row atoms.

\section{Acknowledgements}

The authors would like to thank Prof. M. E. Casida for helpful comments on an early draft of this paper. A. H. and R. M. acknowledge funding by the Deutsche Forschungsgemeinschaft (DFG SPP 1391 Ultrafast Nanooptics MI-1236/3-2). R. M. acknowledges the support by the ERC Consolidator Grant DYNAMO (Grant Nr. 646737)

\section{A Different approximations for the $\gamma_{A B}$ matrix}

Reproducing DFTB results requires some care not only due to the different parametrizations but also because the various implementations employ slightly different approximations. One such ambiguity concerns the definition of the $\gamma$-matrix:

$$
\gamma_{A B}=\iint F_{A}\left(\left|\vec{r}_{1}-\vec{R}_{A}\right|\right) \frac{1}{\left|\vec{r}_{1}-\vec{r}_{2}\right|} F_{B}\left(\left|\vec{r}_{2}-\vec{R}_{B}\right|\right) d^{3} r_{1} d^{3} r_{2}
$$

where $R=\left|\vec{R}_{A}-\vec{R}_{B}\right|$.

The $\gamma$-matrix describes the change of the Coulomb energy due to charge redistribution between the atoms $A$ and $B$. Charge fluctuations are assumed to be spherically symmetric around each atom but the exact functional form is unknown. Slater and Gaussian functions are obvious candidates, 
since the Coulomb integrals are well-known for these functions. In some DFTB-implementations, the $\gamma$-matrix is based on Slater functions (presumably DFTB+ and the older code by Seifert) while in others it is based on Gaussian functions (Hotbit ${ }^{3}$ ).

\section{A.1 Slater functions}

If the charge fluctuation around an atomic center $A$ is modelled by a Slater function,

$$
F_{A}\left(\left|\vec{r}-\vec{R}_{A}\right|\right)=\frac{\tau_{A}^{3}}{8 \pi} \exp \left(-\tau_{A}\left|\vec{r}-\vec{R}_{A}\right|\right)
$$

the Coulomb integral between two such charge distributions separated by a distance $R=\left|\vec{R}_{A}-\vec{R}_{B}\right|$ reads:

$$
\begin{array}{r}
\gamma_{A B}=\frac{1}{R}\left[1+\frac{\tau_{B}^{4}\left(\tau_{B}^{2}\left(2+\tau_{A} R\right)-\tau_{A}^{2}\left(6+\tau_{A} R\right)\right)}{2\left(\tau_{A}^{2}-\tau_{B}^{2}\right)^{3}} e^{-\tau_{A} R}\right. \\
\left.-\frac{\tau_{A}^{4}\left(\tau_{A}^{2}\left(2+\tau_{B} R\right)-\tau_{B}^{2}\left(6+\tau_{B} R\right)\right)}{2\left(\tau_{A}^{2}-\tau_{B}^{2}\right)^{3}} e^{-\tau_{B} R}\right]
\end{array}
$$

In the limit, that the charge distributions are centered at the same position, the $\gamma$-matrix becomes:

$$
\lim _{R \rightarrow 0} \gamma_{A B}=\frac{\tau_{A} \tau_{B}\left(\tau_{A}^{2}+3 \tau_{A} \tau_{B}+\tau_{B}^{2}\right)}{2\left(\tau_{A}^{2}+\tau_{B}^{2}\right)^{3}}
$$

The width parameter $\tau_{A}$ is fixed by the requirement $\gamma_{A A}(R=0)=U_{A}$ :

$$
\tau_{A}=\frac{16}{5} U_{A}
$$

If both atoms are of the same type, one finds:

$$
\lim _{\tau_{A} \rightarrow \tau_{B}=\tau} \gamma_{A B}(R)=\frac{1}{R}\left[1-\frac{48+33(\tau R)+9(\tau R)^{2}+(\tau R)^{3}}{48} e^{-\tau R}\right]
$$




\section{A.2 Gaussian functions}

In the case of Gaussian functions, the charge distribution is modelled by

$$
F_{A}\left(\left|\vec{r}-\vec{R}_{A}\right|\right)=\frac{1}{\left(2 \pi \sigma_{A}^{2}\right)^{3 / 2}} \exp \left(-\frac{\left(\vec{r}-\vec{R}_{A}\right)^{2}}{2 \sigma_{A}^{2}}\right)
$$

Again, the Coulomb integral between two Gaussian charge fluctuations can be performed analytically, yielding:

$$
\gamma_{A B}=\frac{\operatorname{erf}\left(C_{A B} R\right)}{R}
$$

with

$$
C_{A B}=\frac{1}{\sqrt{2\left(\sigma_{A}^{2}+\sigma_{B}^{2}\right)}}
$$

$\sigma_{A}$ is determined by

$$
\lim _{R \rightarrow 0} \gamma_{A A}=\lim _{R \rightarrow 0} \frac{\operatorname{erf}\left(\frac{R}{2 \sigma_{A}}\right)}{R}=\frac{1}{\sqrt{\pi} \sigma_{A}} \stackrel{!}{=} U_{A}
$$

which leads to

$$
\sigma_{A}=\frac{1}{\sqrt{\pi} U_{A}}
$$

for the width parameter.

\section{B Long-range switching functions and the $R_{A B} \rightarrow 0$ limit}

Our long-range correction for DFTB has been criticized for neglecting the short range correction to the exchange energy and using an approximate $\gamma_{A B}^{l r}$ that has the supposedly wrong $R_{A B} \rightarrow 0$ limit. Both issues are related and we would like to defend our method by the following argument:

The error function is not the only and probably not the best way to separate the Coulomb potential into a short and a long range part. For a general switching function $f(r)$ the separation 
becomes:

$$
\frac{1}{r}=\underbrace{\frac{1-f(r)}{r}}_{\text {short range }}+\underbrace{\frac{f(r)}{r}}_{\text {long range }}
$$

Iikura's LC-functionals ${ }^{25}$ and Niehaus' range-separated DFTB employ the error function

$$
f_{\text {erf }}(r)=\operatorname{erf}(\omega r) \quad \text { with } \omega=\frac{1}{R_{\mathrm{lr}}}
$$

But there are other choices. Toulouse ${ }^{24}$ has shown that adding a Gaussian to the switching function (termed erfgau)

$$
f_{\text {erfgau }}(r)=\operatorname{erf}(\omega r)-\frac{2}{\sqrt{\pi}}(\omega r) \exp \left(-\frac{1}{3}(\omega r)^{2}\right)
$$

has the advantage that the correction to the exchange functional that needs to be added to compensate the presence of the long-range HF exchange at short distance is reduced. An expansion of the exchange functional around $\omega=0$,

$$
E_{x, \text { erfgau }}^{s r}=E_{x}+O\left(\omega^{5}\right)
$$

demonstrates that the correction is negligible for $\omega<1$.

The limit of the long-range $\gamma$-matrix for $R_{A B} \rightarrow 0$ depends also on the switching function. We approximate this matrix as:

$$
\begin{aligned}
\gamma_{A B}^{\mathrm{lr}} & =\iint F_{A}\left(\left|\vec{r}_{1}-\vec{R}_{A}\right|\right) \frac{f\left(r_{12}\right)}{r_{12}} F_{B}\left(\left|\vec{r}_{2}-\vec{R}_{B}\right|\right) d^{3} r_{1} d^{3} r_{2} \\
& \approx f\left(R_{A B}\right) \iint F_{A}\left(\left|\vec{r}_{1}-\vec{R}_{A}\right|\right) \frac{1}{r_{12}} F_{B}\left(\left|\vec{r}_{2}-\vec{R}_{B}\right|\right) d^{3} r_{1} d^{3} r_{2} \\
& =f\left(R_{A B}\right) \gamma_{A B}
\end{aligned}
$$

One can argue that the approximation is not warranted, since for erf the numerical solution of the integral leads to a maximum for $R_{A B} \rightarrow 0$ (see Fig. 1 in reference ${ }^{12}$ ), while our approximation 
gives 0 . However, if erfgau is used as a switching function the integral approaches a minimum in the limit $R_{A B} \rightarrow 0$. The shape of $\gamma^{l r}\left(R_{A B}\right)$ at small distance depends strongly on the choice of the switching function, while the large distance limit is not affected.

We proceed by computing $\gamma^{l r}$ numerically for the erf and erfgau switching functions and compare the numerically exact results with the approximation in eqn. ??. It should become clear that the short range behaviour is immaterial and that it is actually beneficial that the long-range correction vanishes for $R_{A B} \rightarrow 0$, as then the short-range modifications to the exchange functional (or the error incurred by neglecting them) are smaller.

Assuming a spherical distribution

$$
F_{A}\left(\left|\vec{r}-\vec{R}_{A}\right|\right)=\frac{\tau_{A}^{2}}{8 \pi} \exp \left(-\tau_{A}\left|\vec{r}-\vec{R}_{A}\right|\right)
$$

the integral can be transformed into a one-dimensional integral (see Niehaus ${ }^{12}$ )

$$
\gamma_{A B}^{\mathrm{lr}}=\frac{\tau_{A}^{4} \tau_{B}^{4}}{\pi R_{A B}} \frac{1}{\mathrm{i}} \int_{0}^{\infty} \frac{\sin \left(q R_{A B}\right)}{\left(q^{2}+\tau_{A}^{2}\right)^{2}\left(q^{2}+\tau_{B}^{2}\right)^{2}} \tilde{f}(q) d q
$$

where $\tilde{f}(q)$ is the Fourier transform of the switching function:

$$
\tilde{f}(q)=\int_{-\infty}^{\infty} f(r) e^{\mathrm{i} q r} d r \quad \text { assuming } f(-r)=-f(r)
$$

The Fourier transforms of the two switching functions are

$$
\tilde{f}_{\text {erf }}(q)=\frac{2 \mathrm{i}}{q} \exp \left(-\frac{q^{2}}{4 \omega^{2}}\right)
$$

and

$$
\tilde{f}_{\text {erfgau }}(q)=\frac{2 \mathrm{i}}{q} \exp \left(-\frac{q^{2}}{4 \omega^{2}}\right)-\frac{3^{3 / 2} \mathrm{i}}{\omega^{2}} q \exp \left(-3 \frac{q^{2}}{4 \omega^{2}}\right)
$$

In Fig. 8 the switching functions and $\gamma^{l r}$-integrals are compared. The short distance behaviour is completely different for erf and erfgau. 
erf

a)

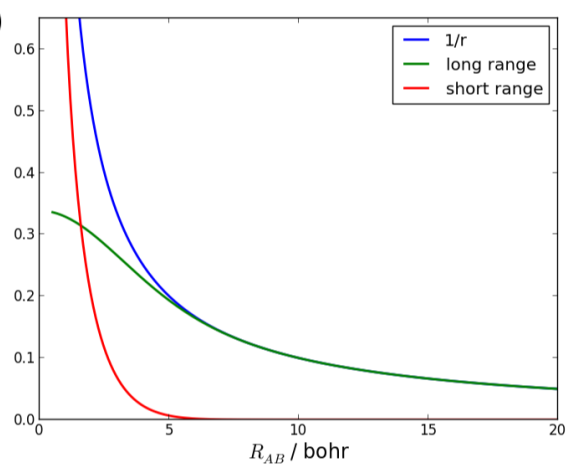

C)

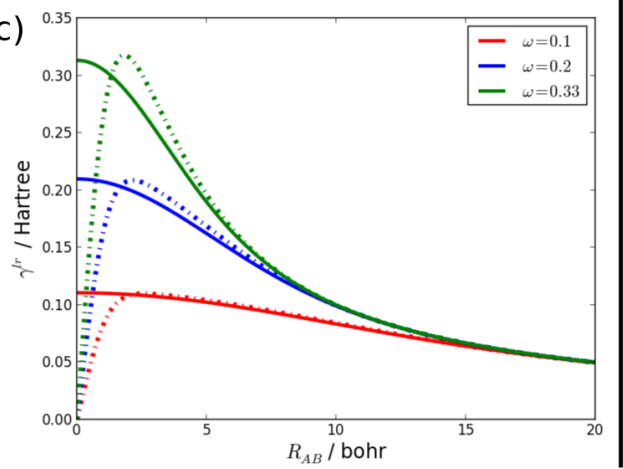

erfgau
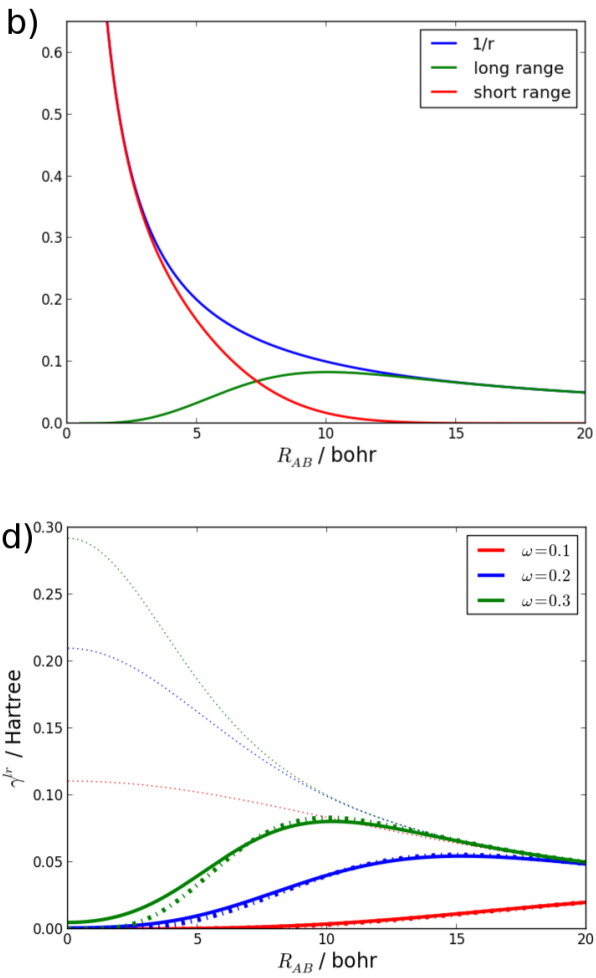

Figure 8: In a) and b) the separation of the Coulomb potential into a short and long range part is depicted for the switching functions erf and erfgau, respectively. c) and d) show the resulting distance dependence of $\gamma^{l r}$ for the two switching functions. The dashed lines show the approximation according to eqn. ?? and the solid lines show the numerically exact integrals ??. The thin lines in d) show the erf-integrals for comparison.

\section{Precalculated dipole matrix elements}

The dipole matrix element between two atomic orbitals $\mu$ and $v$ can be rewritten, so that it depends on the relative position $\vec{R}_{\mu \nu}=\vec{R}_{\nu}-\vec{R}_{\mu}$ of the two centers and the overlap of the two orbitals:

$$
\begin{aligned}
\left\langle\mu\left(\vec{r}-\vec{R}_{\mu}\right)|\vec{r}|\right. & \left.v\left(\vec{r}-\vec{R}_{v}\right)\right\rangle=\int d^{3} r \phi_{\mu}^{*}\left(\vec{r}-\vec{R}_{\mu}\right) \vec{r} \phi_{v}\left(\vec{r}-\vec{R}_{v}\right) \\
\stackrel{\vec{r}^{\prime}}{ }=\vec{r}_{-}-\vec{R}_{\mu} & \int d^{3} r^{\prime} \phi_{\mu}^{*}\left(\vec{r}^{\prime}\right)\left(\vec{r}^{\prime}+\vec{R}_{\mu}\right) \phi_{v}\left(\vec{r}^{\prime}+\vec{R}_{\mu}-\vec{R}_{v}\right) \\
& \left.=\int d^{3} r^{\prime} \phi_{\mu}^{*}\left(\vec{r}^{\prime}\right) \vec{r}^{\prime} \phi_{v}\left(\vec{r}^{\prime}-\vec{R}_{\mu v}\right)+\vec{R}_{\mu} \int d r^{\prime 3} \phi_{\mu}^{*}\left(\vec{r}^{\prime}\right) \phi_{v}\left(\vec{r}^{\prime}-\vec{R}_{\mu v}\right)\right)
\end{aligned}
$$

The second summand in the last line of eqn. ?? can be calculated from Slater-Koster rules for 
the overlap matrix elements between orbitals $\mu$ and $v$

$$
\vec{R}_{\mu} \int d^{3} r^{\prime} \phi_{\mu}^{*}\left(\vec{r}^{\prime}\right) \phi_{v}\left(\vec{r}^{\prime}-\vec{R}_{\mu v}\right)=\vec{R}_{\mu} S_{\mu v}\left(\vec{R}_{\mu v}\right)
$$

whereas the first summand needs special treatment as it contains the dipole operator. The Cartesian components of the dipole operator can be written in terms of $p_{x}, p_{y}$ and $p_{z}$ orbitals located at the origin:

$$
\begin{aligned}
x & =\sqrt{\frac{4 \pi}{3}} r p_{x} \\
y & =\sqrt{\frac{4 \pi}{3}} r p_{y} \\
z & =\sqrt{\frac{4 \pi}{3}} r p_{z}
\end{aligned}
$$

Therefore the dipole matrix element can be understood as the overlap of three orbitals at two centers:

- the orbital $\mu$ at the origin,

- a vector of p-orbitals representing the direction of the position operator, also centered at the origin

- and the orbital $v$ at the center $\vec{R}_{\mu \nu}=\vec{R}_{\nu}-\vec{R}_{\mu}$.

The atomic orbitals consist of a radial part $R_{n l}(r)$ and an angular part $\tilde{Y}_{l, m}(\theta, \phi)$ :

$$
\phi_{\mu}(\vec{r})=R_{n_{\mu} l_{\mu}}(r) \tilde{Y}_{l_{\mu}, m_{\mu}}(\theta, \phi)
$$

The angular parts are real spherical harmonics: 


\begin{tabular}{c|c|c|c} 
Orbital & $l$ & $m$ & $\tilde{Y}_{l m}(\theta, \phi)$ \\
\hline$s$ & 0 & 0 & $\frac{1}{2 \sqrt{\pi}}$ \\
$p_{y}$ & 1 & -1 & $\frac{1}{2} \sqrt{\frac{3}{\pi}} \sin (\theta) \sin (\phi)$ \\
$p_{z}$ & 1 & 0 & $\frac{1}{2} \sqrt{\frac{3}{\pi}} \cos (\theta)$ \\
$p_{x}$ & 1 & 1 & $\frac{1}{2} \sqrt{\frac{3}{\pi}} \sin (\theta) \cos (\phi)$
\end{tabular}

The radial part $R_{n l}(r)$ is specific to each atom type and is obtained by numerically solving the radial Schrödinger equation for the atomic Kohn-Sham Hamiltonian with a local exchange correlation functional. For the valence $s$ - and $p_{x}, p_{y}$ and $p_{z}$ orbitals $(l, m)$ would take the values $(0,0),(1,1),(1,-1)$ and $(1,0)$ respectively. The valence shell of carbon, for instance, requires two radial functions, $R_{n=2, l=0}^{\mathrm{C}}(r)$ for the $2 \mathrm{~s}$ orbital, and $R_{n=2, l=1}^{\mathrm{C}}(r)$ for the three $2 \mathrm{p}$ orbitals.

Decomposing the orbitals $\mu$ and $v$ into their radial and angular parts and expressing the dipole operator by eqns.7374 and 75, the integral for the first summand in eqn. ?? becomes (in spherical coordinates):

$$
\begin{aligned}
& \int d^{3} r^{\prime} \phi_{\mu}^{*}\left(\vec{r}^{\prime}\right) \vec{r}^{\prime} \phi_{v}\left(\vec{r}^{\prime}-\vec{R}_{\mu v}\right)=\underbrace{\int_{0}^{\infty} r_{1}^{2} d r_{1} \int_{0}^{\pi} \sin \left(\theta_{1}\right) d \theta_{1} \int_{0}^{2 \pi} d \phi_{1}}_{d^{3} r_{1}} \\
& \times \underbrace{R_{n_{\mu} l_{\mu}}^{*}\left(r_{1}\right) \tilde{Y}_{l_{\mu} m_{\mu}}^{*}\left(\theta_{1}, \phi_{1}\right)}_{\phi_{\mu}^{*}\left(\vec{r}_{1}\right)} \underbrace{\sqrt{\frac{4 \pi}{3} r_{1}\left(\begin{array}{c}
\tilde{Y}_{1,1}\left(\theta_{1}, \phi_{1}\right) \\
\tilde{Y}_{1,-1}\left(\theta_{1}, \phi_{1}\right) \\
\tilde{Y}_{1,0}\left(\theta_{1}, \phi_{1}\right)
\end{array}\right)} \underbrace{R_{n_{v} l_{v}}\left(\left|\vec{r}_{1}-\vec{R}_{\mu v}\right|\right) \tilde{Y}_{l_{v} m_{v}}\left(\theta_{2}, \phi_{2}\right)}_{\phi_{v}\left(\vec{r}_{2}\right)}}_{\vec{r}_{1}}
\end{aligned}
$$

where $r_{2}=\left|\vec{r}_{1}-\vec{R}_{\mu v}\right|, \theta_{2}$ and $\phi_{2}$ depend on the integration variables $r_{1}, \theta_{1}$ and $\phi_{1}$ as illustrated in Fig.9a).

We wish to find a way to precalculate these integrals and tabulate them, so that at runtime no integrals have to be solved. At first it seems, as if one had to solve the integral for all possible relative arrangement in 3D space (expressed by $\vec{R}_{\mu \nu}$ ) of the two orbitals. Slater-Koster rules $\frac{48}{\text { allow }}$ to break the integral down to a set of few elementary integrals, that only depend on the relative 
distance, and from which the integrals for any relative orientation can be assembled quickly.

To derive the Slater-Koster rules for dipole matrix elements, we begin by rotating the coordinate system such that $\vec{R}_{\mu v}$ points along the $z$ axis. Since spherical harmonics form a representation of the rotation group $S O(3)$ for each angular momentum $l$, the action of this rotation is to mix spherical harmonics with different $m$ but the same $l$. For (complex) spherical harmonics the mixing is described by the Wigner D-matrices, analogously real spherical harmonics, as they are used for orbitals, will be transformed by combinations of those $D$-matrices, which will be called $\tilde{D}$ matrices:

$$
\mathscr{R}_{\vec{R}_{\mu v} \rightarrow \hat{z}}\left[\tilde{Y}_{l m}(\theta, \phi)\right]=\sum_{m^{\prime}=-l}^{l} \tilde{D}_{m, m^{\prime}}^{l}(A, B, \Gamma) \tilde{Y}_{l, m^{\prime}}(\boldsymbol{\theta}, \phi)
$$

The $\tilde{D}$ matrices are expressed as functions of three Euler angles $A, B$ and $\Gamma$. In order to align the vector $\vec{R}_{\mu v}$ (whose spherical coordinates are $R, \Theta$ and $\Phi$ ) with the z-axis, the angles have to be set to $A=\frac{\pi}{2}, B=\Theta$ and $\Gamma=\Phi$. In the integral ?? all three spherical harmonics have to be rotated leading to:

$$
\begin{aligned}
\int d^{3} r^{\prime} \phi_{\mu}^{*}\left(\vec{r}^{\prime}\right) \vec{r}^{\prime} \phi_{v}\left(\vec{r}^{\prime}-\vec{R}_{\mu v}\right)=\sum_{m_{1}=-l_{\mu}}^{l_{\mu}} \sum_{m_{2}=-1}^{1} \sum_{m_{3}=-l_{v}}^{l_{v}} \underbrace{\left(\tilde{D}_{m_{\mu}, m_{1}}^{l_{\mu}}\right)^{*}\left(\begin{array}{c}
\tilde{D}_{1, m_{2}}^{1} \\
\tilde{D}_{-1, m_{2}}^{1} \\
\tilde{D}_{0, m_{2}}^{0}
\end{array}\right) \tilde{D}_{m_{v}, m_{3}}^{l_{v}}}_{T_{i\left(l_{\mu}, l_{v}, m_{\mu}, m_{v}, m_{1}, m_{2}, m_{3}\right)}} \\
\times \underbrace{\int_{0}^{\infty} r_{1}^{2} d r_{1} \int_{0}^{\pi} \sin \left(\theta_{1}\right) d \theta_{1} r_{1} R_{n_{\mu}, l_{\mu}}^{*}\left(r_{1}\right) R_{n_{v}, l_{v}}\left(r_{2}\right)}_{\phi_{i\left(l_{\mu}, l_{v}, m_{1}, m_{2}, m_{3}\right)}\left(\theta_{1}, \theta_{2}\right)} \\
\times \underbrace{\sqrt{\frac{4 \pi}{3}} \int_{0}^{2 \pi} d \phi \tilde{Y}_{l_{\mu}, m_{1}}^{*}\left(\theta_{1}, \phi\right) \tilde{Y}_{1, m_{2}}\left(\theta_{1}, \phi\right) \tilde{Y}_{l_{v}, m_{3}}\left(\theta_{2}, \phi\right)}
\end{aligned}
$$

The rotated coordinate systems and mixing of spherical harmonics is depicted in Fig.9p) and c).

Since after the rotation the z-axes for both orbital centered coordinate systems coincide, one has $\phi_{1}=\phi_{2}$ and the $\phi$ integral can be done analytically. ${ }^{49}$ For $s$ and $p$ orbitals 8 different expressions $\phi_{i}\left(\theta_{1}, \theta_{2}\right)$ result, which are listed in the appendix $\mathrm{D}$ in table 6. The remaining two-dimensional integrals over $r_{1}$ and $\theta_{1}$ are best performed in cylindrical coordinates $\rho, z$. The variable transfor- 
mations are given by

$$
\begin{aligned}
& r_{1}=\sqrt{\rho^{2}+(z-h)^{2}} \\
& r_{2}=\sqrt{\rho^{2}+(z+h)^{2}} \\
& \theta_{1}=\arctan 2(\rho, z-h) \\
& \theta_{2}=\arctan 2(\rho, z+h)
\end{aligned}
$$

where $h=\frac{R_{\mu v}}{2}$ is half the distance between the two atomic centers (see Fig.9d) ). Since the orbitals are highly peaked at the atomic position and decay exponentially towards larger distances, a grid with sampling points clustered around the two atomic centers (see Fig. 9e) ) is most suited for accurate quadrature.

In total, one has to calculate the 8 two-dimensional integrals $D_{i}\left(R_{\mu \nu}\right)$ listed in the third column of table 6 as a function of the orbital separation and save them to a file. The coefficients $T_{i}(x, y, z)$ depend on the directional cosines of the vector $\vec{R}_{\mu v}$, i.e. $x=\cos (\alpha), y=\cos (\beta)$ and $z=\cos (\gamma)$ as labelled in Fig.9a), and account for the relative orientation of the orbitals.

$$
\int d^{3} r^{\prime} \phi_{\mu}^{*}\left(\vec{r}^{\prime}\right) \vec{r}^{\prime} \phi_{v}\left(\vec{r}^{\prime}-\vec{R}_{\mu v}\right)=\sum_{i} T_{i}(x, y, z) D_{i}\left(R_{\mu v}\right)
$$

The Slater-Koster rules for computing $\sum_{i} T_{i} D_{i}$ are given in table 7 of the appendix $\mathrm{E}$. The 3 integrals needed for dipole matrix elements between the valence orbitals of carbon and hydrogen are shown in Fig. 10 as a function of the interatomic distance. 


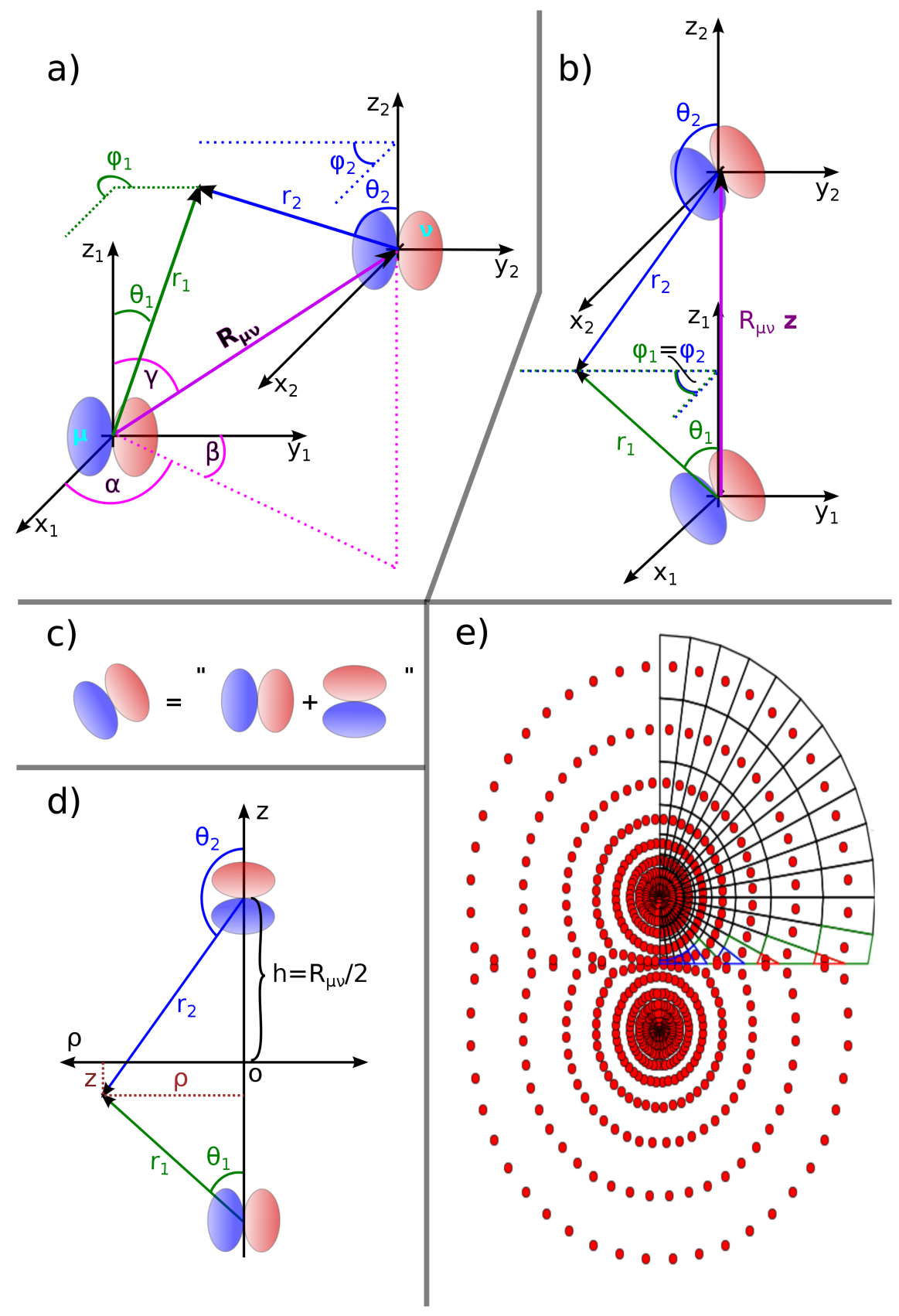

Figure 9: a) Local coordinate systems 1 and 2 around orbitals $\mu$ and $v$. The spherical coordinates of a position $\vec{r}^{\prime}$ can be specified with respect to either axis. The direction of the vector $\vec{R}_{\mu \nu}$ joining the two orbital centers is defined by the directional cosines $x=\cos (\alpha), y=\cos (\beta)$ and $z=\cos (\gamma)$. b) After rotating the coordinate systems $\vec{R}_{\mu \nu}$ coincides with the z-axes. c) The rotated spherical harmonics are linear combinations of spherical harmonics aligned with the axes. d) Cylindrical coordinates. e) Grid for integration. Two polar grids centered at the atomic positions are merged for an efficient distribution of sampling points around both atoms (implementation of Hotbit ${ }^{3}$ ). The plane is divided into rectangles or triangles, whose size increases away from each center. 


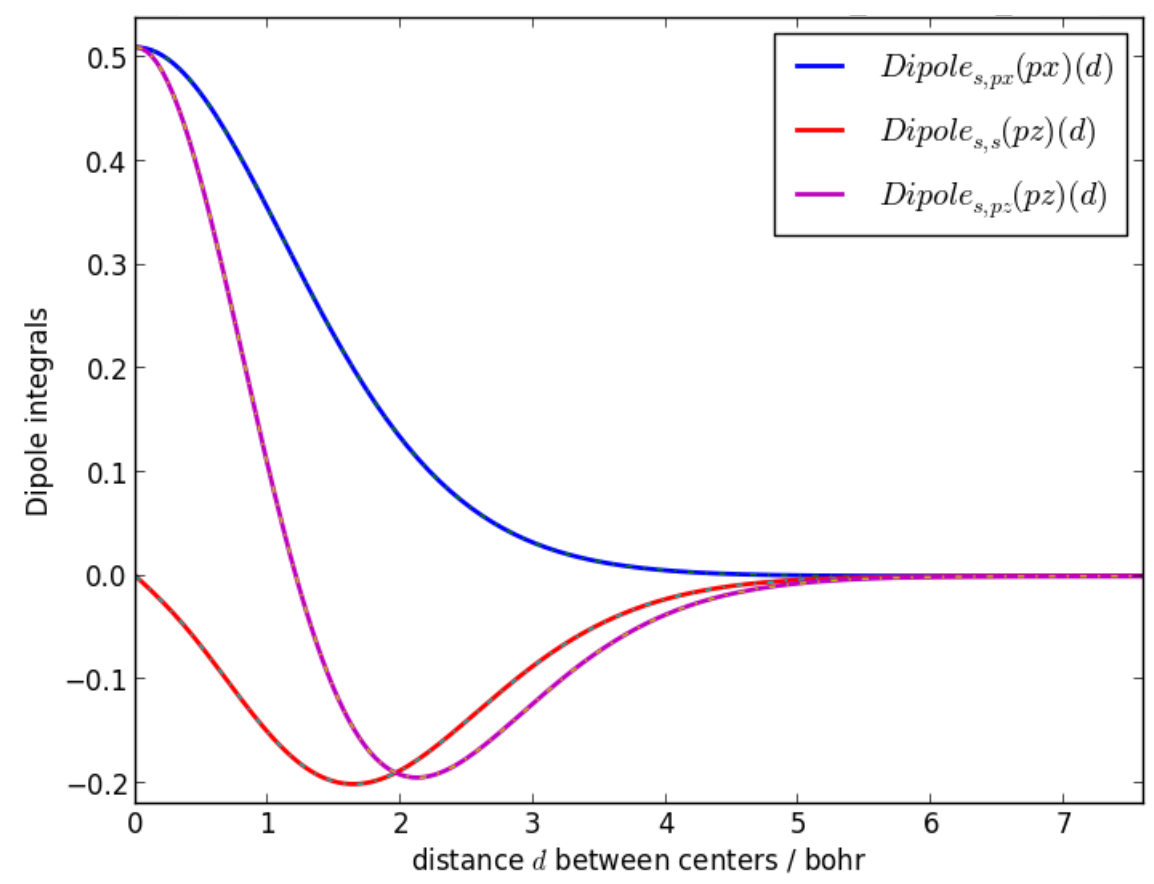

Figure 10: Precalculated dipole integrals for the atom pair h-c that are tabulated in Slater-Koster files, $D_{1}$ (blue), $D_{2}$ (red) and $D_{3}$ (violet).

\section{Unique radial integrals $D_{i}$}

Table 6: List of the angular and radial integrals. $R_{n_{\mu}, l_{\mu}}(r)$ denotes the radial function with angular momentum $l_{\mu}$ for the orbital $\mu$ of one atom type. $r_{1}, r_{2}$ and $\theta_{1}$ and $\theta_{2}$ depend on the cylindrical integration variables $\rho$ and $z$ as explained in eqns. 80.83 .

\begin{tabular}{c|c|c}
$i$ & $\phi_{i}\left(\theta_{1}, \theta_{2}\right)$ & Integrals $D_{i}\left(R_{\mu v}\right)$ \\
\hline 1 & $\frac{3}{8 \sqrt{\pi}} \sin \left(\theta_{1}\right) \sin \left(\theta_{2}\right)$ & $\iint d z \rho d \rho R_{n_{\mu}, l_{\mu}=0}\left(r_{1}\right) r_{1} R_{n_{v}, l_{v}=1}\left(r_{2}\right) \phi_{1}\left(\theta_{1}, \theta_{2}\right)$ \\
2 & $\frac{\sqrt{3}}{4 \sqrt{\pi}} \cos \left(\theta_{1}\right)$ & $\iint d z \rho d \rho R_{n_{\mu}, l_{\mu}=0}\left(r_{1}\right) r_{1} R_{n_{v}, l_{v}=0}\left(r_{2}\right) \phi_{1}\left(\theta_{1}, \theta_{2}\right)$ \\
3 & $\frac{3}{4 \sqrt{\pi}} \cos \left(\theta_{1}\right) \cos \left(\theta_{2}\right)$ & $\iint d z \rho d \rho R_{n_{\mu}, l_{\mu}=0}\left(r_{1}\right) r_{1} R_{n_{v}, l_{v}=1}\left(r_{2}\right) \phi_{1}\left(\theta_{1}, \theta_{2}\right)$ \\
4 & $\frac{3}{8 \sqrt{\pi}} \sin ^{2}\left(\theta_{1}\right)$ & $\iint d z \rho d \rho R_{n_{\mu}, l_{\mu}=1}\left(r_{1}\right) r_{1} R_{n_{v}, l_{v}=0}\left(r_{2}\right) \phi_{1}\left(\theta_{1}, \theta_{2}\right)$ \\
5 & $\frac{3}{8} \sqrt{\frac{3}{\pi}} \sin ^{2}\left(\theta_{1}\right) \cos \left(\theta_{2}\right)$ & $\iint d z \rho d \rho R_{n_{\mu}, l_{\mu}=1}\left(r_{1}\right) r_{1} R_{n_{v}, l_{v}=1}\left(r_{2}\right) \phi_{1}\left(\theta_{1}, \theta_{2}\right)$ \\
6 & $\frac{3}{8} \sqrt{\frac{3}{\pi}} \sin \left(\theta_{1}\right) \cos \left(\theta_{1}\right) \sin \left(\theta_{2}\right)$ & $\iint d z \rho d \rho R_{n_{\mu}, l_{\mu}=1}\left(r_{1}\right) r_{1} R_{n_{v}, l_{v}=1}\left(r_{2}\right) \phi_{1}\left(\theta_{1}, \theta_{2}\right)$ \\
7 & $\frac{3}{4 \sqrt{\pi}} \cos ^{2}\left(\theta_{1}\right)$ & $\iint d z \rho d \rho R_{n_{\mu}, l_{\mu}=1}\left(r_{1}\right) r_{1} R_{n_{v}, l_{v}=0}\left(r_{2}\right) \phi_{1}\left(\theta_{1}, \theta_{2}\right)$ \\
8 & $\frac{3 \sqrt{3}}{4 \sqrt{\pi}} \cos ^{2}\left(\theta_{1}\right) \cos \left(\theta_{2}\right)$ & $\iint d z \rho d \rho R_{n_{\mu}, l_{\mu}=1}\left(r_{1}\right) r_{1} R_{n_{v}, l_{v}=1}\left(r_{2}\right) \phi_{1}\left(\theta_{1}, \theta_{2}\right)$
\end{tabular}




\section{E Slater-Koster rules for assembling dipole matrix elements}

Table 7 summarizes the rules for calculating dipole matrix elements between two atomic-centered orbitals from Slater-Koster tables for the integrals $D_{i}\left(\vec{R}_{12}\right)$. The atomic orbital $\phi_{1}$ is centered on an atom at position $\vec{R}_{1}$ and the second atomic orbital $\phi_{2}$ is centered on another atom at position $\vec{R}_{2}$. $x, y$ and $z$ are the directional cosines for the vector $\vec{R}_{12}$ pointing from the first to the second center. The 8 integrals $D_{i=1, \ldots, 8}(r)$ are precalculated for all distances $r=\left|\vec{R}_{12}\right|$ and tabulated. So far only $s$ - and $p$-orbital are considered. Since the rules were obtained from a computer algebra program written for the software package Mathematica, $\underline{50}$ rules for $d$-orbitals could also be obtained easily. 
Table 7: Slater-Koster rules for dipole matrix elements (for s and p-orbitals). For clarity the distance between orbital centers is named $r$ instead of $R_{\mu \nu}$.

\begin{tabular}{|c|c|c|c|}
\hline orb. $\phi_{1}$ & coord. & orb. $\phi_{2}$ & Rule for $\left\langle\phi_{1}\left(\vec{r}-\vec{R}_{1}\right)\left|\left(\vec{r}-\vec{R}_{12}\right)\right| \phi_{2}\left(\vec{r}-\vec{R}_{2}\right)\right\rangle$ \\
\hline$s$ & $\mathrm{y}$ & $s$ & $y D_{2}(r)$ \\
\hline$s$ & $\mathrm{y}$ & $p_{y}$ & $\left(x^{2}+z^{2}\right) D_{1}(r)+y^{2} D_{3}(r)$ \\
\hline$s$ & $\mathrm{y}$ & $p_{z}$ & $y z\left(D_{3}(r)-D_{1}(r)\right)$ \\
\hline$s$ & $\mathrm{y}$ & $p_{x}$ & $x y\left(D_{3}(r)-D_{1}(r)\right)$ \\
\hline$s$ & $\mathrm{z}$ & $s$ & $z D_{2}(r)$ \\
\hline$s$ & $\mathrm{z}$ & $p_{y}$ & $y z\left(D_{3}(r)-D_{1}(r)\right)$ \\
\hline$s$ & $\mathrm{z}$ & $p_{z}$ & $\left(x^{2}+y^{2}\right) D_{1}(r)+z^{2} D_{3}(r)$ \\
\hline$s$ & $\mathrm{z}$ & $p_{x}$ & $x z\left(D_{3}(r)-D_{1}(r)\right)$ \\
\hline$s$ & $\mathrm{x}$ & $s$ & $x D_{2}(r)$ \\
\hline$s$ & $\mathrm{x}$ & $p_{y}$ & $x y\left(D_{3}(r)-D_{1}(r)\right)$ \\
\hline$s$ & $\mathrm{x}$ & $p_{z}$ & $x z\left(D_{3}(r)-D_{1}(r)\right)$ \\
\hline$s$ & $\mathrm{x}$ & $p_{x}$ & $\left(y^{2}+z^{2}\right) D_{1}(r)+x^{2} D_{3}(r)$ \\
\hline$p_{y}$ & $\mathrm{y}$ & $s$ & $\left(x^{2}+y^{2}\right) D_{4}(r)+y^{2} D_{7}(r)$ \\
\hline$p_{y}$ & $\mathrm{y}$ & $p_{y}$ & $y\left(x^{2}+z^{2}\right)\left(D_{5}(r)+2 D_{6}(r)\right)+y^{3} D_{8}(r)$ \\
\hline$p_{y}$ & $\mathrm{y}$ & $p_{z}$ & $z\left(\left(x^{2}+z^{2}\right) D_{5}(r)+y^{2}\left(D_{8}(r)-2 D_{6}(r)\right)\right)$ \\
\hline$p_{y}$ & $\mathrm{y}$ & $p_{x}$ & $x\left(\left(x^{2}+z^{2}\right) D_{5}(r)+y^{2}\left(D_{8}(r)-2 D_{6}(r)\right)\right)$ \\
\hline$p_{y}$ & $\mathrm{z}$ & $s$ & $y z\left(D_{7}(r)-D_{4}(r)\right)$ \\
\hline$p_{y}$ & $\mathrm{z}$ & $p_{y}$ & $z\left(\left(x^{2}-y^{2}+z^{2}\right) D_{6}(r)+y^{2}\left(D_{8}(r)-D_{5}(r)\right)\right)$ \\
\hline$p_{y}$ & $\mathrm{z}$ & $p_{z}$ & $y\left(\left(x^{2}+y^{2}\right) D_{6}(r)-z^{2}\left(D_{5}(r)+D_{6}(r)-D_{8}(r)\right)\right)$ \\
\hline$p_{y}$ & $\mathrm{z}$ & $p_{x}$ & $x y z\left(D_{8}(r)-D_{5}(r)-2 D_{6}(r)\right)$ \\
\hline$p_{y}$ & $\mathrm{x}$ & $s$ & $x y\left(D_{7}(r)-D_{4}(r)\right)$ \\
\hline$p_{y}$ & $\mathrm{x}$ & $p_{y}$ & $x\left(\left(x^{2}-y^{2}+z^{2}\right) D_{6}(r)+y^{2} D_{8}(r)-y^{2} D_{5}(r)\right)$ \\
\hline$p_{y}$ & $\mathrm{x}$ & $p_{z}$ & $x y z\left(D_{8}(r)-D_{5}(r)-2 D_{6}(r)\right)$ \\
\hline$p_{y}$ & $\mathrm{x}$ & $p_{x}$ & $y\left(x^{2}\left(D_{8}(r)-D_{5}(r)\right)+\left(z^{2}-x^{2}+y^{2}\right) D_{6}(r)\right)$ \\
\hline$p_{z}$ & $\mathrm{y}$ & $s$ & $y z\left(D_{7}(r)-D_{4}(r)\right)$ \\
\hline$p_{z}$ & $\mathrm{y}$ & $p_{y}$ & $z\left(y^{2}\left(D_{8}(r)-D_{5}(r)\right)+\left(x^{2}-y^{2}+z^{2}\right) D_{6}(r)\right)$ \\
\hline$p_{z}$ & $\mathrm{y}$ & $p_{z}$ & $y\left(\left(x^{2}+y^{2}\right) D_{6}(r)-z^{2}\left(D_{5}(r)+D_{6}(r)-D_{8}(r)\right)\right)$ \\
\hline$p_{z}$ & $\mathrm{y}$ & $p_{x}$ & $x y z\left(D_{8}(r)-D_{5}(r)-2 D_{6}(r)\right)$ \\
\hline$p_{z}$ & $\mathrm{z}$ & $s$ & $\left(x^{2}+y^{2}\right) D_{4}(r)+z^{2} D_{7}(r)$ \\
\hline$p_{z}$ & $\mathrm{z}$ & $p_{y}$ & $y\left(\left(x^{2}+y^{2}\right) D_{5}(r)+z^{2}\left(D_{8}(r)-2 D_{6}(r)\right)\right)$ \\
\hline$p_{z}$ & $\mathrm{z}$ & $p_{z}$ & $\left(x^{2}+y^{2}\right) z\left(D_{5}(r)+2 D_{6}(r)\right)+z^{3} D_{8}(r)$ \\
\hline$p_{z}$ & $\mathrm{z}$ & $p_{x}$ & $x\left(\left(x^{2}+y^{2}\right) D_{5}(r)+z^{2}\left(D_{8}(r)-2 D_{6}(r)\right)\right)$ \\
\hline$p_{z}$ & $\mathrm{x}$ & $s$ & $x z\left(D_{7}(r)-D_{4}(r)\right)$ \\
\hline$p_{z}$ & $\mathrm{x}$ & $p_{y}$ & $x y z\left(D_{8}(r)-D_{5}(r)-2 D_{6}(r)\right)$ \\
\hline$p_{z}$ & $\mathrm{x}$ & $p_{z}$ & $x\left(\left(x^{2}+y^{2}\right) D_{6}(r)-z^{2}\left(D_{5}(r)+D_{6}(r)-D_{8}(r)\right)\right)$ \\
\hline$p_{z}$ & $\mathrm{x}$ & $p_{x}$ & $z\left(x^{2}\left(D_{8}(r)-D_{5}(r)\right)+\left(y^{2}-x^{2}+z^{2}\right) D_{6}(r)\right)$ \\
\hline$p_{x}$ & $\mathrm{y}$ & $s$ & $x y\left(D_{7}(r)-D 4(r)\right)$ \\
\hline$p_{x}$ & $\mathrm{y}$ & $p_{y}$ & $x\left(y^{2}\left(D_{8}(r)-D_{5}(r)\right)+\left(x^{2}-y^{2}+z^{2}\right) D_{6}(r)\right)$ \\
\hline$p_{x}$ & $\mathrm{y}$ & $p_{z}$ & $x y z\left(D_{8}(r)-D_{5}(r)-2 D_{6}(r)\right)$ \\
\hline$p_{x}$ & $\mathrm{y}$ & $p_{x}$ & $y\left(x^{2}\left(D_{8}(r)-D_{5}(r)\right)+\left(y^{2}-x^{2}+z^{2}\right) D_{6}(r)\right)$ \\
\hline$p_{x}$ & $\mathrm{z}$ & $s$ & $x z\left(D_{7}(r)-D_{4}(r)\right)$ \\
\hline$p_{x}$ & $\mathrm{z}$ & $p_{y}$ & $x y z\left(D_{8}(r)-D_{5}(r)-2 D_{6}(r)\right)$ \\
\hline$p_{x}$ & $\mathrm{z}$ & $p_{z}$ & $x\left(\left(x^{2}+y^{2}\right) D_{6}(r)-z^{2}\left(D_{5}(r)+D_{6}(r)-D_{8}(r)\right)\right)$ \\
\hline$p_{x}$ & $\mathrm{z}$ & $p_{x}$ & $z\left(x^{2}\left(D_{8}(r)-D_{5}(r)\right)+\left(y^{2}-x^{2}+z^{2}\right) D_{6}(r)\right)$ \\
\hline$p_{x}$ & $\mathrm{x}$ & $s$ & $\left(y^{2}+z^{2}\right) D_{4}(r)+x^{2} D_{7}(r)$ \\
\hline$p_{x}$ & $\mathrm{x}$ & $p_{y}$ & $y\left(\left(y^{2}+z^{2}\right) D_{5}(r)+x^{2}\left(D_{8}(r)-2 D_{6}(r)\right)\right)$ \\
\hline$p_{x}$ & $\mathrm{x}$ & $p_{z}$ & $z\left(\left(y^{2}+z^{2}\right) D_{5}(r)+x^{2}\left(D_{8}(r)-2 D_{6}(r)\right)\right)$ \\
\hline$p_{x}$ & $\mathrm{x}$ & $p_{x}$ & $x\left(y^{2}+z^{2}\right)\left(D_{5}(r)+2 D_{6}(r)\right)+x^{3} D_{8}(r)$ \\
\hline
\end{tabular}




\section{F Analysing charge transfer with density differences}

Different "metrics" have been devised to identify charge-transfer states automatically and warn about possible failures of TD-DFT. Since the $\Lambda$-metric ${ }^{1}$ cannot detect all problematic charge transfer excitations, Guido et.al. introduced the $\Delta r \underline{51}$ - and $\Gamma^{[52}$-metrics. In particular the $\Delta r$-metric has an intuitive interpretation as the electron-hole distance.

We can define a similar quantity at the tight-binding level. To this end, we start with the density difference between the excited state and the ground state, $\Delta \rho_{I}=\rho_{I}-\rho_{0}$. In the linear response regime the Kohn-Sham "wavefunction" of the excited state $I$ is a linear combination of single excitations from the Kohn-Sham ground state Slater determinant:

$$
\left|\Psi_{I}\right\rangle=\sum_{v \in \operatorname{virt} o \in \text { occ }} C_{v o}^{I} \hat{a}_{v}^{\dagger} \hat{a}_{o}\left|\Psi_{0}\right\rangle
$$

The operator for the electron density in second quantization reads (in the basis of Kohn-Sham orbitals):

$$
\hat{\rho}(\vec{r})=\sum_{\alpha} \sum_{\beta} \hat{a}_{\alpha}^{\dagger} \hat{a}_{\beta} \phi_{\alpha}^{*}(\vec{r}) \phi_{\beta}(\vec{r})
$$

Here, $o$ and $o^{\prime}$ denote occupied, $v$ and $v^{\prime}$ virtual and $\alpha$ and $\beta$ general Kohn-Sham orbitals. Combining eqns. ?? and ??, the density of state $I$ becomes:

$$
\rho_{I}(\vec{r})=\left\langle\Psi_{I}|\hat{\rho}| \Psi_{I}\right\rangle=\sum_{o, o^{\prime}} \sum_{v, v^{\prime}} \sum_{\alpha, \beta} \phi_{\alpha}^{*}(\vec{r}) \phi_{\beta}(\vec{r}) C_{v o}^{I *} C_{v^{\prime} o^{\prime}}^{I}\left\langle\Psi_{0}\left|\hat{a}_{o}^{\dagger} \hat{a}_{v} \hat{a}_{\alpha}^{\dagger} \hat{a}_{\beta} \hat{a}_{v^{\prime}}^{\dagger} \hat{a}_{o^{\prime}}\right| \Psi_{0}\right\rangle
$$

By using anti-commutation relations for Fermions, and the fact that the ground state only contains

occupied orbitals (so $\hat{a}_{v}\left|\Psi_{0}\right\rangle=\hat{a}_{o}^{\dagger}\left|\Psi_{0}\right\rangle=0$ ), the expression for the density can be reduced to: $\frac{53}{53}$

$$
\begin{aligned}
\rho_{I}(\vec{r}) & =\sum_{o} \sum_{v, v^{\prime}} C_{v o}^{I *} C_{v^{\prime} o}^{I} \phi_{v}^{*}(\vec{r}) \phi_{v}(\vec{r})-\sum_{v} \sum_{o, o^{\prime}} C_{v o}^{I *} C_{v o^{\prime}}^{I} \phi_{o^{\prime}}^{*}(\vec{r}) \phi_{o}(\vec{r})+\sum_{o}\left|\phi_{o}(\vec{r})\right|^{2} \\
& =\rho_{p}(\vec{r})+\rho_{h}(\vec{r})+\rho_{o}(\vec{r})
\end{aligned}
$$


In the exciton picture, the $I$-th excited state can be described by a bound particle-hole pair. The first term in eqn. 88 can be identified with the particle, the second term belongs to the hole, while the last term is just the ground state density. The difference density between the ground and excited state is the sum of the particle and hole densities:

$$
\Delta \rho_{I}=\rho_{I}-\rho_{0}=\rho_{e}+\rho_{h}
$$

Since tight-binding DFT deals with partial charges instead of a continuous density distribution, we have to coarse grain the density to an atomic resolution. Again, the transition charges are approximated as a sum of spherically symmetric charge distributions centered on the individual atoms:

$$
\begin{aligned}
\phi_{v}^{*}(\vec{r}) \phi_{v^{\prime}}(\vec{r}) & =\sum_{A} q_{A}^{v v^{\prime}} F_{A}\left(\left|\vec{r}-\vec{R}_{A}\right|\right) \\
\phi_{o}^{*}(\vec{r}) \phi_{o^{\prime}}(\vec{r}) & =\sum_{A} q_{A}^{o o^{\prime}} F_{A}\left(\left|\vec{r}-\vec{R}_{A}\right|\right)
\end{aligned}
$$

The particle and hole densities are partitioned into atomic contributions,

$$
\begin{aligned}
& \rho_{e}=\sum_{A} \sum_{o, v, v^{\prime}} C_{v o}^{I *} C_{v^{\prime} o}^{I} q_{A}^{v v^{\prime}} F_{A}\left(\left|\vec{r}-\vec{R}_{A}\right|\right)=\sum_{A} q_{A}^{e} F_{A}\left(\left|\vec{r}-\vec{R}_{A}\right|\right) \\
& \rho_{h}=\sum_{A} \sum_{v, o, o^{\prime}} C_{v o}^{I *} C_{v o^{\prime}}^{I} q_{A}^{o o^{\prime}} F_{A}\left(\left|\vec{r}-\vec{R}_{A}\right|\right)=\sum_{A} q_{A}^{h} F_{A}\left(\left|\vec{r}-\vec{R}_{A}\right|\right)
\end{aligned}
$$

$q_{A}^{e}$ and $q_{A}^{h}$ are the particle-hole charges.

The average positions of the particle and the hole result from the weighted average of all charges.

$$
\begin{aligned}
& \vec{r}_{e}=\frac{\sum_{A} q_{A}^{e} \vec{R}_{A}}{\sum_{A} q_{A}^{e}} \\
& \vec{r}_{h}=\frac{\sum_{A} q_{A}^{h} \vec{R}_{A}}{\sum_{A} q_{A}^{h}}
\end{aligned}
$$


The particle-hole separation $d_{e-h}=\left|\vec{r}_{e}-\vec{r}_{h}\right|$ indicates the spatial extent of the exciton. Fig 11 shows that in the $\beta$-dipeptide, absorption of a photon can lead to a separation of positive and negative charge over a distance of 10.0 bohr.

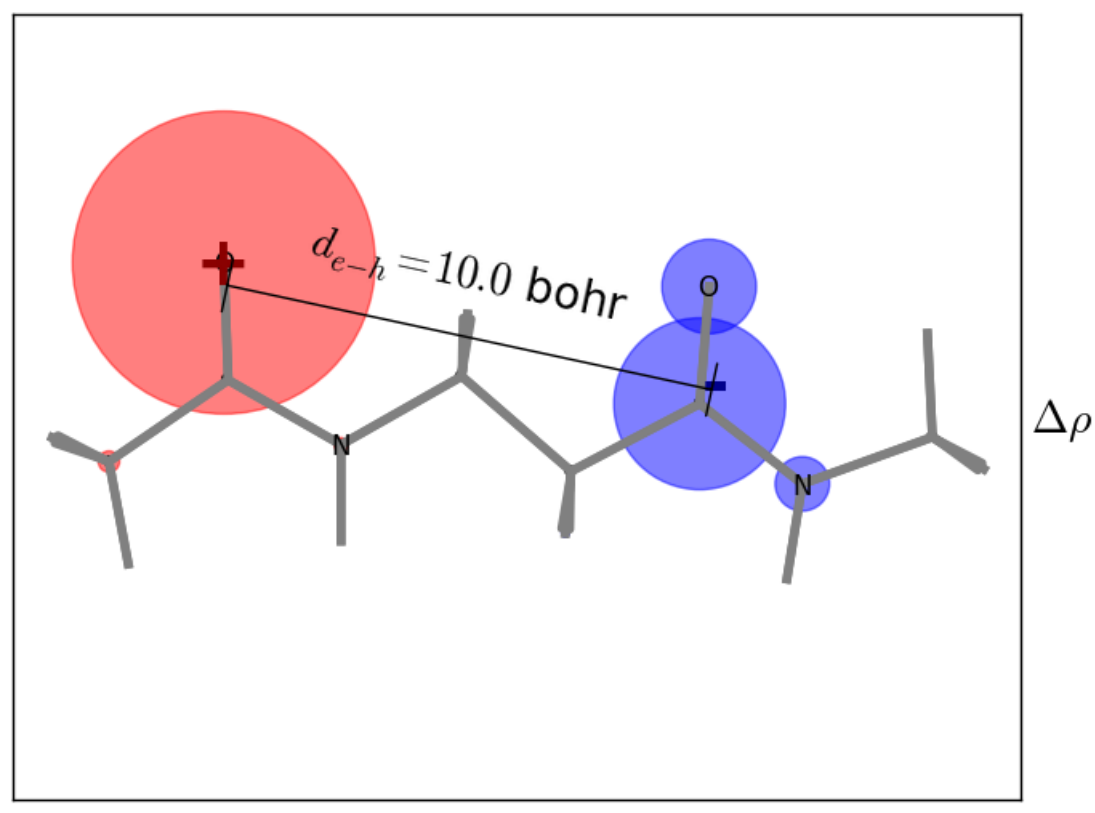

Figure 11: Difference density $\Delta \rho$ for the 3rd excited state of the $\beta$-dipeptide. An exciton is formed as an electron jumps from the first carbonyl group to the second leaving a positive hole behind. The radii of the red (blue) circles are proportional to the hole (particle) charges on each atom.

\section{G Supporting Information}

A more extensive assessment of the quality of lc-TD-DFTB excitation energies is given in the Supporting Information. This material is available free of charge via the Internet at http://pubs.acs.org

\section{References}

(1) Peach, M.; Benfield, P.; Helgaker, T.; Tozer, D.; Excitation energies in density functional theory: An evaluation and a diagnostic test. J. Chem. Phys. 2008, 128, 044118. 
(2) Elstner, M.; Porezag, D.; Jungnickel, G.; Elsner, J.; Haugk, M.; Fraunheim, Th.; Suhai, S.; Seifert, G.; Self-consistent-charge density-functional tight-binding method for simulations of complex materials properties. Phys. Rev. B, 1998, 58, 7260-7268.

(3) Koskinen, P.; Mäkinen, V.; Density-functional tight-binding for beginners. Computational Materials Science, 2009, 24, 237-253.

(4) Elstner, M.; Fraunheim, Th.; Kaxiras, E.; Seifert, G.; Suhai, S.; A Self-Consistent Charge Density-Functional Based Tight-Binding Scheme for Large Biomolecules. Phys. Stat. Sol. B, 2000, $217,357$.

(5) Niehaus, T.; Suhai, S.; Della Sala, F.; Lugli, R.; Elstner, M.; Seifert, G.; Fraunheim, T.; Tight-binding approach to time-dependent density-functional response theory. Phys. Rev. B, 2001, 63, 085108.

(6) Trani, F.; Scalmani, G.; Zheng, G.; Carnimeo, I.; Frisch, M.; Barone, V.; Time-Dependent Density Functional Tight Binding: New Formulation and Benchmark of Excited States. $J$. Chem. Theory Comput., 2011, 7, 3304-3313.

(7) Casida, M.; Time-dependent density-functional response theory for molecules. D.P. Chong (Ed.), Recent Advances in Density Functional Methods Part I, World Scientific, Singapore 1995, p. 155.

(8) Casida, M.; Time-dependent density-functional theory for molecules and molecular solids Journal of Molecular Structure, 2009, THEOCHEM 914, 3-18.

(9) Furche, F.; Ahlrichs, R.; Adiabatic time-dependent density functional methods for excited state properties. J. Chem. Phys., 2002, 117, 7433-7447.

(10) Magyar, R.; Dependence of Spurious Charge-Transfer Excited States on Orbital Exchange in TDDFT: Large Molecules and Clusters. J. Chem. Theory Comput., 2007, 3, 976-987. 
(11) Tawada, Y.; Tsuneda, T.; Yanagisawa, S.; Yanai, T.; Hirao, K.; A long-range-corrected time-dependent density functional theory. J. Chem. Phys. 2004, 120, 8425-8433.

(12) Niehaus, T.; Della Sala, F.; Range separated functionals in the density functional based tightbinding method: Formalism. Phys. Status Solidi B, 2012, 2, 237-244.

(13) Yanai, T.; Tew, D.; Handy,N.; A new hybrid exchange-correlation functional using the Coulomb-attenuating method (CAM-B3LYP). Chem. Phys. Lett., 2004, 393, 51-57.

(14) Oliveira, A.; Seifert, G.; Heine, Th.; Duarte, H.; Density-Functional Based Tight-Binding: an Approximate DFT Method. J. Braz. Chem. Soc., 2009, 20, 1193-1205.

(15) Elstner, M.; Seifert, G. Density functional tight binding. Phil. Trans. R. Soc. A, 2014, 372, 20120483.

(16) Kohn, W.; Sham, L.; Self-consistent equations including exchange and correlation effects. Phys. Rev., 1965, 140, 1133-1138.

(17) Gaus, M.; Goez, A.; Elstner, M.; Parametrization and Benchmark of DFTB3 for Organic Molecules. J. Chem. Theory Comput., 2013, 9, 338-354.

(18) Krüger, Th.; Elstner, M.; Validation of the density-functional based tight-binding approximation method for the calculation of reaction energies and other data. J. Chem. Phys., 2005, $122,114110$.

(19) Dreuw, A.; Head-Gordon, M.; Single-Reference ab Initio Methods for the Calculation of Excited States of Large Molecules. Chem. Rev., 2005, 105, 4009-4037.

(20) Isborn, Ch.; Luehr, N.; Ufimtsev, I.; Martínez, T.; Excited-State Electronic Structure with Configuration Interaction Singles and Tamm Dancoff Time-Dependent Density Functional Theory on Graphical Processing Units. J. Chem. Theory Comput., 2011, 7, 1814-1823. 
(21) Cordova, F.; Doriol, J.; Ipatov, A.; Casida, M.; Troubleshooting Time-Dependent DensityFunctional Thoery for Photochemical Applications: Oxirane. J. Chem. Phys., 2007, 127, 164111.

(22) Peach, M.; Tozer, D.; Overcoming Low Orbital Overlap and Triplet Instability Problems in TDDFT. J. Phys. Chem. A, 2012, 116, 9783-9789.

(23) Stratmann, R.; Scuseria, G.; Frisch, M.; An efficient implementation of time-dependent density-functional theory for the calculation of excitation energies of large molecules. $J$. Chem. Phys., 1998, 109, 8218-8224.

(24) Toulouse, J.; Colonna, F.; Savin, A.; Long-range-short-range separation of the electronelectron interaction in density-functional theory Phys. Rev. A, 2004, 70, 062505.

(25) Iikura, H.; Tsuneda, T.; Yanai, T.; Hirao, K.; A long-range correction scheme for generalized-gradient-approximation exchange functionals. J. Chem. Phys., 2001, 115, 35403544.

(26) Jorgensen, P.; Molecular and Atomic Applications of Time-Dependent Hartree-Fock Theory. Annu. Rev. Phys. Chem., 1975, 26, 359-380.

(27) Domínguez, A.; Aradi, B.; Fraunheim, T.; Lutsker, V.; Niehaus, T.; Extensions of the TimeDependent Density Functional Based Tight-Binding Approach. J. Chem. Theory Comput., 2013, 9, 4901-4914.

(28) Becke, A.; Density-functional thermochemistry. III. The role of exact exchange. J. Chem. Phys., 1993, 98, 5648-5652.

(29) Lee, C.; Yang, W.; Parr, R.; Development of the Colle-Salvetti correlation-energy formula into a functional of the electron-density. Phys. Rev. B, 1988, 37, 785.

(30) Dunning, Jr.; Gaussian-basis sets for use in correlated molecular calculations. 1. The atoms boron through neon and hydrogen. J. Chem. Phys., 1989, 90, 1007. 
(31) Perdew, J.; Burke,K.; Ernzerhof, M.; Generalized gradient approximation made simple. Phys. Rev. Lett., 1996, 77, 3865-3868.

(32) Stephens, P.; Devlin, F.; Chabalowski, C.; Ab Initio Calculation of Vibrational Absorption and Circular Dichroism Spectra Using Density Functional Force Fields. J. Phys. Chem., 1994, 98, 11623-11627.

(33) Perdew, J.; Wan, Y.; Accurate and simple analytic representation of the electron-gas correlation energy. Phys. Rev. B, 1992, 45, 13244.

(34) Thijssen, J.; Computational Physics, 1999, Cambridge University Press.

(35) Kramida, A.; Ralchenko, Yu.; Reader, J. and NIST ASD Team (2014).; NIST Atomic Spectra Database (ver. 5.2), [Online]. Available: http://physics.nist.gov/asd [2014, October 21]. National Institute of Standards and Technology, Gaithersburg, MD.

(36) Rienstra-Kiracofe, J.; Tschumper, G.; Schaefer III, F.; Atomic and Molecular Electron Affinities: Photoelectron Experiments and Theoretical Computations. Chem. Rev., 2002, 102, 231-282.

(37) Cordero, B.; Gómez, V.; Platero-Prats, A.; Revés, M.; Echeverría, J.; Cremades, E.; Barragán, F.; Alvarez, S.; Covalent radii revisited. Dalton Trans., 2008, 2832-2838.

(38) Tozer, D.; see http://community.dur.ac.uk/d.j.tozer/benchmark.html

(39) Gaussian 09, Revision D.01, M. J. Frisch, G. W. Trucks, H. B. Schlegel, G. E. Scuseria, M. A. Robb, J. R. Cheeseman, G. Scalmani, V. Barone, B. Mennucci, G. A. Petersson, H. Nakatsuji, M. Caricato, X. Li, H. P. Hratchian, A. F. Izmaylov, J. Bloino, G. Zheng, J. L. Sonnenberg, M. Hada, M. Ehara, K. Toyota, R. Fukuda, J. Hasegawa, M. Ishida, T. Nakajima, Y. Honda, O. Kitao, H. Nakai, T. Vreven, J. A. Montgomery, Jr., J. E. Peralta, F. Ogliaro, M. Bearpark, J. J. Heyd, E. Brothers, K. N. Kudin, V. N. Staroverov, R. Kobayashi, J. Normand, K. Raghavachari, A. Rendell, J. C. Burant, S. S. Iyengar, J. Tomasi, M. Cossi, N. Rega, 
J. M. Millam, M. Klene, J. E. Knox, J. B. Cross, V. Bakken, C. Adamo, J. Jaramillo, R. Gomperts, R. E. Stratmann, O. Yazyev, A. J. Austin, R. Cammi, C. Pomelli, J. W. Ochterski, R. L. Martin, K. Morokuma, V. G. Zakrzewski, G. A. Voth, P. Salvador, J. J. Dannenberg, S. Dapprich, A. D. Daniels, Ö. Farkas, J. B. Foresman, J. V. Ortiz, J. Cioslowski, and D. J. Fox, Gaussian, Inc., Wallingford CT, 2009.

(40) Rettig, W.; Zietz, B.; Do twisting and pyramidalization contribute to the reaction coordinate of charge-transfer formation in DMABN and derivatives? Chem. Phys. Lett., 2000, 317, 187-196.

(41) Manz, J.; Proppe, B.; Schmidt, B.; Time-resolved dual fluorescence of 1-phenylpyrrole in acetonitrile: molecular dynamics simulations of solvent response to twisted intramolecular charge transfer. Phys. Chem. Chem. Phys., 2002, 4, 1876-1881.

(42) Wiggins, P.; Williams, G.; Tozer, D.; Excited state surfaces in density functional theory: A new twist on an old problem. J. Chem. Phys, 2005, 122, 084314.

(43) Hehre, W.; Stewart, R.; Pople, J.; Self-consistent molecular-orbital methods. I. Use of Gaussian expansions of Slater-type atomic orbitals. J. Chem. Phys., 1969, 2657.

(44) Dreuw, A.; Weisman, J.; Head-Gordon, M.; Long-range charge-transfer excited states in time-dependent density functional theory require non-local exchange. J. Chem. Phys., 2003, 119, 2943.

(45) Schreiber, M.; Silva-Junior, M.; Sauer, S.; Thiel,W. Benchmarks for electronically excited states: CASPT2, CC2, CCSD and CC3. J. Chem. Phys., 2008, 128, 134110.

(46) Moreira, N.; Dolgonos, G.; Aradi, B.; da Rosa, A.; Fraunheim, Th.; Toward an Accurate Density-Functional Tight-Binding Description of Zinc-Containing Compounds. J. Chem. Theory Comput., 2009, 5, 605-614. 
(47) Wahiduzzaman, M.; Oliveira, A.; Philipsen, P.; Zhechkov, L.; van Lenthe, E.; Witek, H.; Heine, Th.; DFTB Parameters for the Periodic Table: Part 1, Electronic Structure. J. Chem. Theory Comput., 2013, 9, 4006-4017.

(48) Slater, C.; Koster, G.; Simplified LCAO method for the periodic potential problem. Phys. Rev., 1954, 94, 1498.

(49) Edmonds, A.; Angular Momentum in Quantum Mechanics. Angular Momentum in Quantum Mechanics, 1996, Princeton University Press.

(50) Wolfram Research, Inc., Mathematica, Version 10.0, Champaign, IL (2014).

(51) Guido, C.; Cortona, P.; Mennucci, B.; Adamo, C.; On the Metric of Charge Transfer Molecular Excitations: A Simple Chemical Descriptor. J. Chem. Theory Comput., 2013, 9, 31183126.

(52) Guido, C.; Cortona, P.; Adamo, C.; Effective electron displacements: A tool for timedependent density functional theory computational spectroscopy. J. Chem. Phys., 2014, 140, 104101.

(53) Zhang, X.; Li, Z.; Lu, G.; First-principle simulations of exciton diffusion in organic semiconductors. Phys. Rev. B, 2011, 84, 235208. 
Graphical TOC Entry

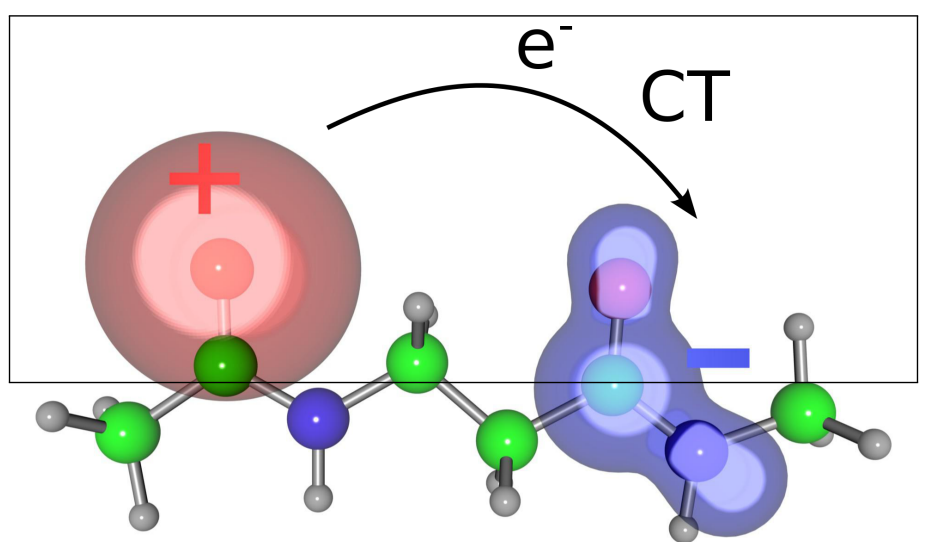

long range corrected DFTB 